Elsevier required licence: ( 2019

This manuscript version is made available under the CC-BY-NC-ND 4.0 license http://creativecommons.org/licenses/by-nc-nd/4.0/

The definitive publisher version is available online at $\underline{10.1016 / j . r e n e n e .2018 .05 .093}$ 


\title{
Research on a Combined Model Based on Linear and Nonlinear Features - A Case Study of Wind Speed Forecasting
}

\author{
Kequan Zhang ${ }^{1}$, Zongxi Qu ${ }^{1,}$ *, Yunxuan Dong ${ }^{2}$, Haiyan Lu ${ }^{3}$, Wennan Leng ${ }^{1}$, \\ Jianzhou Wang ${ }^{4}$, Wenyu Zhang ${ }^{1}$ \\ 1 A key Laboratory of Arid Climatic Change and Reducing Disaster of Gansu \\ Province, College of Atmospheric Sciences, Lanzhou University, Lanzhou 730000, \\ China; zhangkq@lzu.edu.cn; quzx14@1zu.edu.cn; lengwn15@lzu.edu.cn; \\ yuzhang@1zu.edu.cn \\ 2 School of mathematics and statics, Lanzhou University, China; \\ dongyx15@1zu.edu.cn \\ 3 Faculty of Engineering and Information Technology, University of Technology, \\ Sydney, Australia; haiyan.lu@uts.edu.au \\ ${ }^{4}$ School of Statistics, Dongbei University of Finance and Economics, China; \\ wjz@lzu.edu.cn \\ * Correspondence: quzx14@1zu.edu.cn;
}

\begin{abstract}
As one of the most promising sustainable energy sources, wind energy is being paid more attention by the researchers. Because of the volatility and instability of wind speed series, wind power integration faces a severe challenge; thus, an accurate wind energy forecasting plays a key role in smart grid planning and management. However, many traditional forecasting models do not consider the necessity and importance of data preprocessing and neglect the limitation of using a single forecasting model, which leads to poor forecasting accuracy. To solve these problems, a novel combined model based on two linear and four nonlinear forecasting algorithms is proposed to adapt both the linear and nonlinear characteristics of the wind energy time series. In addition, a modified Artificial Fish Swarm Algorithm and Ant Colony Optimization (AFSA-ACO) algorithm is proposed and employed to determine the optimal weight coefficients of the combined models. To verify the forecasting performance of the developed combined model, several experiments were implemented by using ten-minute interval wind speed data in Shandong, China. Then, one-step (ten-minute), three-step (thirty-minute) and five-step (fifty-minute) predictions were conducted. The experimental results indicate that the developed combined model is remarkably superior to all benchmark models for the high precision and stability of wind-speed predictions.
\end{abstract}

Keywords: Wind speed forecasting; Combined model; Artificial fish swarm algorithm; Ant colony optimization.

\section{Introduction}

Wind energy, as an alternative to fossil fuel-generated electricity, has received increasing attention around the world due to its abundance, wide distribution, and economics as a non-polluting type of renewable energy [1]. The global cumulatively installed wind capacity reached approximately $539.58 \mathrm{GW}$ by the end of 2017 , which an annual increase of $9.7 \%$. Furthermore, researchers have noted that there will be a new round of installed wind energy in 2018-2021, and that $22 \%$ of the world's electricity will be supplied by wind energy by 2030 [2]. However, the intermittent 
and stochastic nature of wind speed always poses many technical challenges, such as increasing the cost and reducing the performance and reliability of the wind industry and power systems, especially when wind power is integrated into traditional grid systems. Therefore, to alleviate the abovementioned problems, accurate and reliable information of dynamic wind power is increasingly significant and urgently needed, and it can not only be utilized to control the wind turbine, but also help make decisions on wind power system's management [3].

Over the past several decades, a large number of forecasting models for wind speed have been developed and applied to obtain precise information of wind energy. Generally, based on the time horizons, the research of wind speed prediction can be classified into four types: very short-term forecasting, short-term forecasting, medium-term forecasting and long-term forecasting [4]. According to computational mechanisms, these forecasting technologies can be divided into three general classes: physical methods, statistical approaches and intelligent learning methods [3,5,6].

The physical methods are based mostly on Numeric Weather Prediction (NWP), which usually applies meteorological information such as temperature and pressure, for wind speed forecasting. However, NWP is unsuitable for short-term wind speeds because of its complex calculation processes, high costs and poor performance [7]. Compared with physical methods, statistical and intelligent-learning approaches, which extracts the laws of historical data, can provide more accurate results for short-term wind speed forecasting. Specifically, the statistical methods, also taken as time series models, are widely applied and easily implemented in the forecasting field. However, these algorithms, including the auto-regressive integrated moving average (ARIMA), Markov chain, exponential soothing (ES), and Kalman filtering method, can achieve more accurate short-term wind speed predictions than can physical models, which capture the linear relationship between the past and future performance of a time series [8]. In comparison, intelligent learning algorithms such as the Back Propagation Neural Network (BPNN), Radial Basis Function Neural Network (RBFNN), Generalized Regression Neural Network (GRNN) and Wavelet Neural Network (WNN), which structure a network relationship of input and output information, are widely used in the field of wind speed forecasting and perform better at handling the nonlinear features of wind speed data [9]. For example, Guo et al. [10] developed a forecasting model using BPNN and seasonal exponential adjustments to predict wind speed and eventually obtained good forecasts. Yu et al. [11] successfully exploited an Elman Neural Network (ENN) based on an improved wavelet transform using singular spectrum analysis for wind speed forecasting, resulting in good performance.

Because of the inherent weaknesses of every model and the intermittency, complex fluctuation and the linear and nonlinear characteristics of wind speed series, the abovementioned forecasting approaches cannot always capture the trend of the wind speed, especially in nonlinear time series, which usually leads to poor forecasting performance. Thus, with the aim of achieving higher accuracy levels and 
wider forecast horizons, combined model, that combine the advantages of the single forecasting models, have been developed and widely utilized for wind speed prediction [12]. For example, Wang et al. [13] developed a combined model by applying four artificial neural networks (ANNs), the multi-objective bat algorithm and singular spectrum analysis to forecast a 10-min wind speed. The results indicated that the combined model can improve the prediction performance to some extent. Xiao et al. [14] presented a combined model on the basic of the chaotic particle swarm optimization algorithm to optimize the weight coefficients for wind speed prediction.

However, wind speed series have linear and non-linear characteristics. Hence, to improve the forecasting performance, the linear and nonlinear trends that cause the intermittency and complex fluctuations of wind speed should both be considered in the combined models. To capture the linear and nonlinear traits, both linear models, such as ARIMA and ES, and nonlinear methods, such as BPNN, WNN, ENN should be employed in the combined models. Thus, a novel combined forecasting model based on two linear models, i.e., ES and ARIMA, and four nonlinear methods, i.e., BPNN, GRNN, Wavelet Neural Network (WNN) and ENN, is proposed in this paper. To further study the combined models, many evolutional optimization algorithms are applied to help determine the optimal weights of the combination model. For example, Yang et al. [15] used differential evolution (DE) to optimize the weight coefficients of a combination model to improve forecasting accuracy. Xiao et al. [14] applied the cuckoo search algorithm (CSO) to determine the optimal weights of a combined model. Rahmani et al. [16] developed a hybrid algorithm with ant colony and particle swarm optimization for wind energy forecasting. The ant colony optimization (ACO) algorithm, which is based on the behavior of natural ant colonies, has been widely used in many in many engineering optimization problems. Recently, to improve the performance of the ACO with respect to optimized problems, many new modifications have been proposed. Sen et al. [17] developed a hybrid optimization algorithm, ACO-ABC-HS, to solve the problem of economic dispatch (ED) for a multi-generator system. The hybrid algorithm combines the framework of ACO, artificial bee colony (ABC) and harmonic search (HS) algorithms to find the optimized solution for the system. Patel el al. [18] developed a hybrid ant colony optimization (ACO)/particle swarm optimization (PSO) technique to optimize the multicast tree; the results showed that ACO-PSO outperforms both the ACO and the PSO. To enhance the exploration capacities and improve the local searching ability of the ant colony optimization, a modified version of the AFSA and ACO, i.e., the AFSA-ACO, is proposed in this paper. It has been successfully applied to determine the weight coefficients of the combined model. Compared with the artificial fish swarm algorithm (AFSA) and ACO algorithm, the proposed AFSA-ACO, which has been tested by several benchmark functions, not only enhances the performance of the AFSA and ACO with more optimum solutions and higher convergence rates but also has stronger 
capabilities to solve more complex problems. To conduct a case study, the original wind speed datasets with 10-min intervals from four sites in the wind farms of Penglai, Shandong Province, China, were used to verify the proposed combined forecasting model. Since the original wind-speed series are highly unstable and noisy, the singular-spectrum analysis (SSA) [19] was used for data pre-processing to remove the noise of the original wind-speed data for easy forecasting.

The rest of the paper is organized as follows: Section 2 introduces the related individual forecasting models. The combined model and combined theory are presented in Section 3, where a novel modified optimization algorithm, AFSA-ACO, is also presented. The forecasting results of the proposed combined model and comparisons are discussed in Section 4. An insightful discussion of the developed model is conducted in Section 5. Finally, Section 6 concludes the paper.

List of abbreviations

\begin{tabular}{|c|c|c|c|}
\hline AFSA-AC & $\begin{array}{l}\text { Artificial Fish Swarm } \\
\text { Algorithm and Ant Colony } \\
\text { Optimization }\end{array}$ & ENN & Elman Neural Network \\
\hline AFSA & $\begin{array}{l}\text { Artificial Fish Swarm } \\
\text { Algorithm }\end{array}$ & ES & Exponential Smoothing \\
\hline $\mathrm{ACO}$ & Ant Colony Optimization & WNN & Wavelet Neural Network \\
\hline NWP & $\begin{array}{l}\text { Numerical } \\
\text { Weather-Prediction }\end{array}$ & GA & Genetic Algorithm \\
\hline ARIMA & $\begin{array}{l}\text { Auto Regressive Integrated } \\
\text { Moving Average Model }\end{array}$ & PSO & $\begin{array}{l}\text { Particle Swarm } \\
\text { Optimization }\end{array}$ \\
\hline BPNN & $\begin{array}{l}\text { Back Propagation Neural } \\
\text { Network }\end{array}$ & MSE & Mean Squared Error \\
\hline GRNN & $\begin{array}{l}\text { General Regression Neural } \\
\text { Network }\end{array}$ & $\begin{array}{l}\text { MAE } \\
\text { MAPE }\end{array}$ & $\begin{array}{l}\text { Square Sum of the Error } \\
\text { Average of Absolute Error }\end{array}$ \\
\hline RBFNN & $\begin{array}{l}\text { Radial Basis Function Neural } \\
\text { Network }\end{array}$ & NNCT & $\begin{array}{l}\text { No Negative Constraint } \\
\text { Theory }\end{array}$ \\
\hline $\begin{array}{l}\text { SVM } \\
\text { CS }\end{array}$ & $\begin{array}{l}\text { Support Vector Machine } \\
\text { Cuckoo Search }\end{array}$ & SSA & Singular Spectrum Analysis \\
\hline
\end{tabular}

\section{Individual Forecasting Models}

There are many individual models that can be used to handle the issue of wind speed forecasting and are considered effective. Considering the inherent linear and nonlinear features contained in wind speed data, two linear algorithms and four nonlinear algorithms, which performs better in wind speed forecasting, are used to develop the combined forecasting model with the non-positive constraint theory.

\subsection{Linear algorithms}

The Autoregressive Integrated Moving Average (ARIMA) model and Exponential Smoothing(ES) algorithm are chosen as linear models to capture the linear feature of wind speed data in the developed combined model, which is investigates in a series of experiments [20]. 


\subsubsection{Autoregressive Integrated Moving Average (ARIMA)}

The ARIMA model, developed by Box and Jenkins [21], is one of the most popular and widely used forecasting methods. It consists of three parts: autoregressive (AR), moving average (MA) and integration (I). The details are briefly described as follows:

The AR $(p)$ model is an autoregressive model with order $\mathrm{p}$, which is defined as:

$$
y_{t}=c+a_{1} y_{t-1}+\ldots+a_{p} y_{t-p}+u_{t}
$$

where $a_{1}, \ldots, a_{p}$ are parameters, $u_{t}$ is a random variable as a white noise, and $c$ is constant.

The $\mathrm{MA}(q)$ model is a moving average model with order $q$, which is defined as:

$$
y_{t}=\mu+u_{t}+m_{1} u_{t-1}+\ldots+m_{q} u_{t-q}
$$

where $m_{1}, \ldots, m_{q}$ are parameters, $\mu$ is the expectation of $y_{t}$ and $u_{t}, u_{t-1}, \ldots, u_{t-q}$ are white noise error terms.

The ARMA $(p, q)$ model is combined with $\mathrm{p}$ autoregressive terms and $\mathrm{q}$ moving-average terms, and is defined as:

$$
y_{t}=c+a_{1} y_{t-1}+\ldots+a_{p} y_{t-p}+u_{t}+m_{1} u_{t-1}+\ldots+m_{q} u_{t-q}
$$

When $\operatorname{AR}(p), \operatorname{MA}(q)$ and $\operatorname{ARMA}(p, q)$ are used for data with non-stationarity, the differencing step corresponding to the integration part should be performed to eliminate the non-stationarity of researched data, which is defined as an ARIMA (p, d, q) model.

\subsubsection{Exponential Smoothing (ES)}

The double exponential smoothing model is defined as:

$$
R_{t}^{(2)}=\alpha R_{t}^{(1)}+(1-\alpha) R_{t-1}^{(2)}
$$

where $\alpha$ is the smoothing factor $R_{t}^{(2)}, R_{t-1}^{(2)}$ are the second order exponential smoothing values for stages $t$ and $t-1$, and $R_{t}^{(1)}$ is the first order exponential smoothing value.

The initial value is determined by

$$
R_{0}^{(1)}=R_{0}^{(2)}=X_{0}
$$

where $X_{0}$ is the initial value of the original data.

The forecasting formula of the second exponential smoothing is defined as

$$
R_{t+T}=a_{t}+b_{t} \cdot T
$$

$$
\left\{\begin{array}{c}
a_{t}=2 R_{t}^{(1)}-R_{t}^{(2)} \\
b_{t}=\frac{\alpha}{1-\alpha}\left(R_{t}^{(1)}-R_{t}^{(2)}\right)
\end{array}\right.
$$

where $R_{t+T}$ is the forecasting value of stage $t+T$, and $T$ denotes the forecasting stage from stage $t$. 


\subsection{Nonlinear algorithms}

There are several nonlinear algorithms employed to conduct wind speed forecasting. The Back Propagation Neural Network (BPNN), Generalized Regression Neural Network (GRNN), Wavelet Neural Network (WNN) and Elman Neural Network (ENN), have been adopted as nonlinear algorithms to capture the nonlinear characteristics inherent in wind speed data, which are known to be better for wind speed forecasting with relative accuracy. The construction of each nonlinear models is shown in Fig. 2.

\subsubsection{Back Propagation Neural Network (BPNN)}

The Back-propagation algorithm, proposed in 1986 by Rumelhart et al [22], is one of the most popular neural networks. BPNN is a type of multilayer feed-forward neural network that is widely used, and it is based on the gradient descent method. As shown in Fig. 2, BPNN consists of three types of layers: an input layer, a hidden layer and an output layer.

Definition 1. The process of the back-propagation algorithm is divided into two phases: updating and learning.

$$
\begin{gathered}
w_{i j}(t)=w_{i j}(t-1)-\Delta w_{i j}(t) \\
\Delta w_{i j}(t)=\eta \partial E / \partial w_{i j}(t-1)+\alpha \cdot \Delta w_{i j}(t-1)
\end{gathered}
$$

where $w_{i j}$ is the weight between nodes $i$ and $j, \eta$ is the learning speed, $\alpha$ is the impulse parameter, $t$ is the current iterative steps, and $E$ is the error super curve face.

Definition 2. During the learning phase, the thresholds and weights of the joints between two layers are updated by subsequently back-propagating the errors, minimizing the mean squared error (MSE).

$$
M S E=\frac{1}{2} \sum_{k=1}^{m}\left(Y_{k}-O_{k}\right)^{2}
$$

where $O_{k}$ denotes the output values, and $Y_{k}$ denotes the desired output.

\subsubsection{Generalized Regression Neural Network (GRNN)}

The GRNN model, proposed by Specht [23], is a new type of neural network model. There are four types of layers: the input layer, the pattern layer, the summation layer, and the output layer, as presented in Fig 2.

Definition 3. There is a nonlinear transformation in the pattern layer, which binds input space with pattern space. The relationship between the input neuron and the appropriate response from the pattern layer can be memorized in each neuron in this layer.

Definition 4. The summation layer has two summations: the simple summation $S_{s}$ and the weighted summation $S_{w}$. Then, the summation layer is transmitted to the output layer.

\subsubsection{Wavelet Neural Network (WNN)}

The basic structure of WNN is based on wavelet analysis and is presented in Fig. 2. It substitutes the conventional sigmoid function with a mother wavelet.

Definition 5. Function $f(x)$ can approximately describe a cluster of mother wavelets: 


$$
\hat{f}(x)=\sum_{l=0}^{N} w_{l} \cdot \psi_{l}\left(\frac{x-b_{1}}{a_{1}}\right)
$$

228 where $\hat{f}(x)$ denotes a fitting function, $w_{l}$ denotes a weighting coefficient, and $N$

229 denotes the quantity of mother wavelets.

230

\subsubsection{Elman Neural Network (ENN)}

The ENN model, introduced by Elman, consists of three layers: an input layer, a hidden layer, and an output layer. A simple three-layer ENN is presented in Fig. 2. The difference between ENN and the three-layer feed-forward neural network is that the ENN model has a context layer to respond to the outputs of the hidden layer. The activations of the context layer is [24]

$$
S_{i}(t)=g\left(\sum_{k=1}^{K} V_{i k} S_{k}(t-1)+\sum_{j=1}^{j} W_{i j} l_{j}(t-1)\right)
$$

where $S_{k}(t)$ and $I_{j}(t)$ are the output of the context layers and input layers, respectively; $V_{i k}$ and $W_{i j}$ are the weights; and $g(x)$ is a sigmoid transfer function.

\section{Combined Model}

The combined forecasting method is started with J.M. Bates and C.W.J. Granger in the 1960s. They, proved that using two or more models to forecast results is better than using a single model, that is the limitations of the single forecasting model are overcome by the combined forecasting method [25]. Because both linear and nonlinear characteristics appeared in the wind speed time series, a new combined model is developed in this paper that integrates linear and nonlinear models, non-positive constraint theory and a modified optimization algorithm. The main processes of the proposed combined model are illustrated in Fig. 1. 


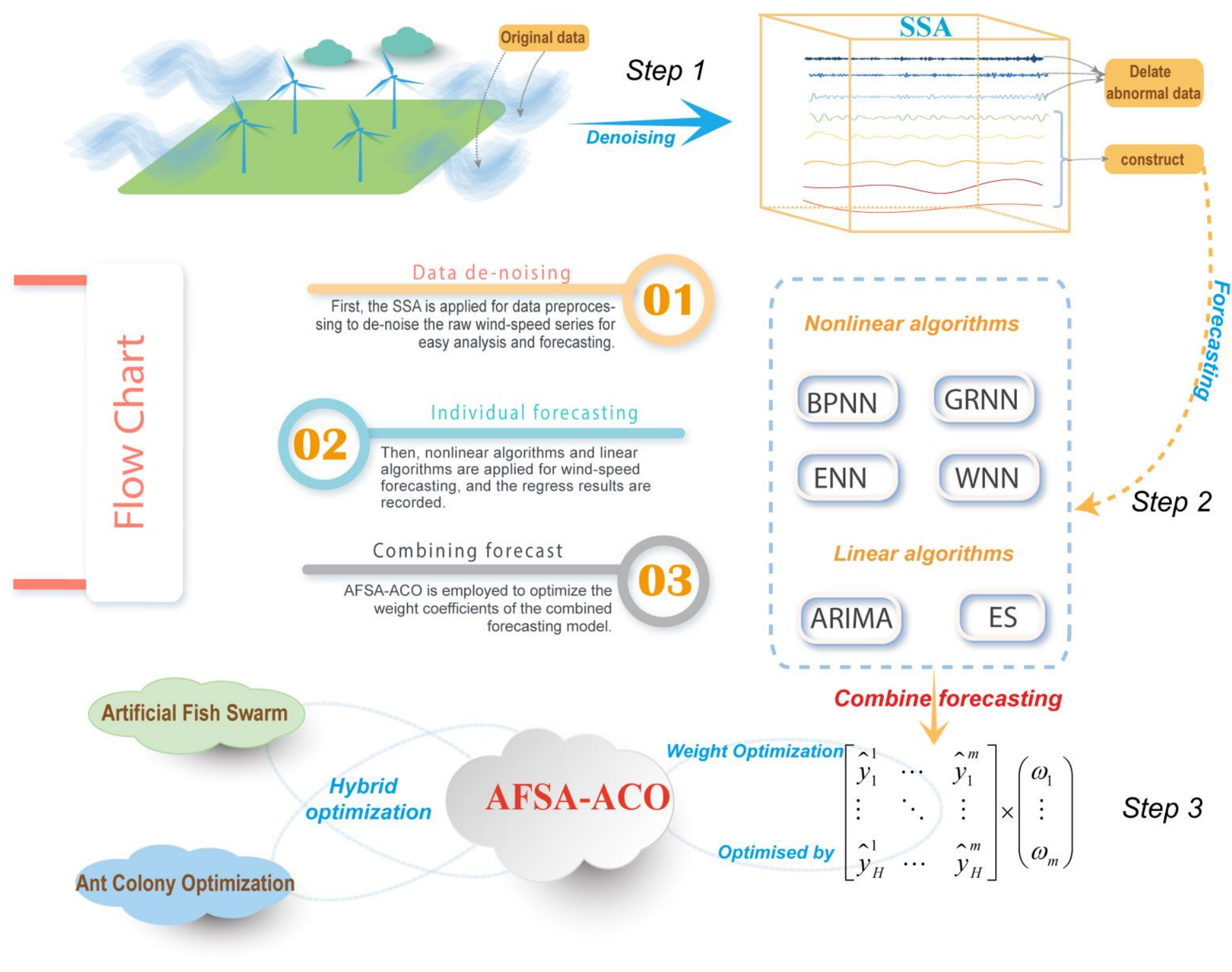


The key of combined forecasting theory is to estimate the weight coefficient. According to an objective function that is contributed by a certain standard, the optimized weight coefficient can be found by minimizing the objective function. The objective function is generally determined by the error value (e.g., Mean Absolute Error (MAE), Mean Square Error (MSE) and Sum of Squares for Error (SSE) et.al) and a series of minimizing standards is explored.

Definition 6. The weight coefficient of the traditional combined forecasting model is calculated by minimizing SSE:

$$
\min \boldsymbol{L}=\boldsymbol{W}^{T} \boldsymbol{E} \boldsymbol{W}=\sum_{t=1}^{T} \sum_{j=1}^{m} \sum_{i=1}^{m} \mathrm{w}_{i} \mathrm{w}_{j} e_{i t} e_{j t}\left\{\begin{array}{c}
\boldsymbol{R}^{T} \boldsymbol{W}=1 \\
\boldsymbol{W} \geq 0
\end{array}\right.
$$

261 where $\boldsymbol{W}=\left(w_{1}, w_{2}, \ldots, w_{m}\right)^{T}$ is the weight vector; $\boldsymbol{R}=(1,1, \ldots, 1)^{T}$ is a column vector and $\boldsymbol{E}=\left(\boldsymbol{E}_{i j}\right)_{m \times m}$ is forecasting error information matrix.

Definition 7. Traditional combination approach based on no negative constraint theory (TCM-NNCT) [14] was developed to improve the traditional method, which is presented as

$$
\min \boldsymbol{L}=\boldsymbol{W}^{T} \boldsymbol{E} \boldsymbol{W}=\sum_{t=1}^{T} \sum_{j=1}^{m} \sum_{i=1}^{m} w_{i} w_{j} e_{i t} e_{j t}
$$

$$
\text { st } \quad \boldsymbol{R}^{T} \boldsymbol{W}=1
$$

For the improved method, TCM-NNCT, the experimental results demonstrated that the combination forecasting model can achieve desirable results when the range of the weight vector $\boldsymbol{W}$ is $[-2,2]$ rather than $[0,1]$. The provided weight-determined approach was tested by experiment instead of relying on theory.

In this paper, a novel combined forecasting model based on TCM-NNCT and weight-coefficient optimization was developed, and contains two linear algorithms (i.e., ES and ARIMA) and four neural network algorithms (i.e., BPNN, GRNN, WNN and ENN), as shown in Fig. 2. 


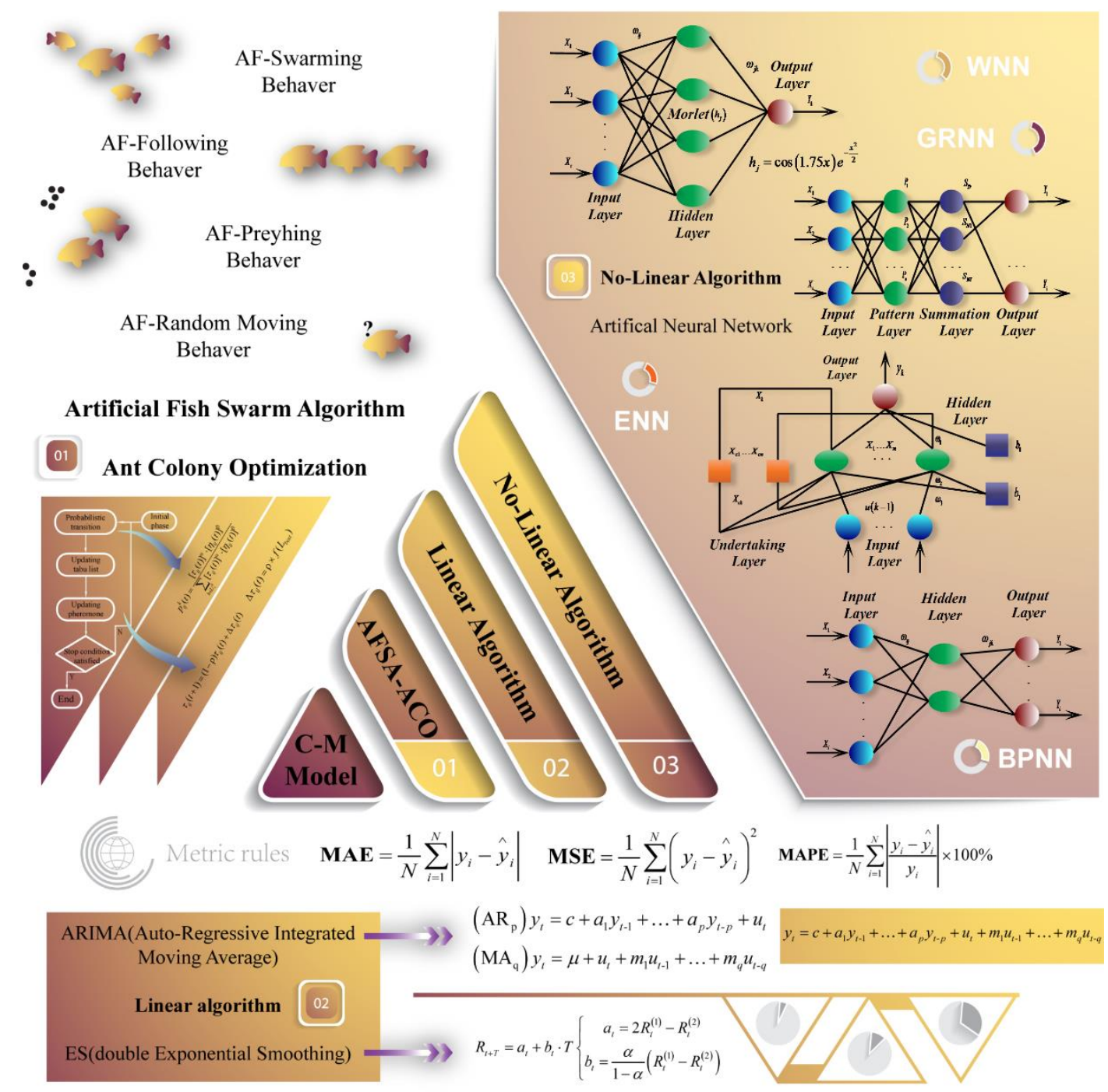

Fig. 2 Structure of the combined models.

\subsection{Optimization algorithm-AFSA-ACO}

To further improve the forecasting performance of the developed model, considering the limitations of ACO in the later optimization period (i.e., the slow convergence speed and the imprecise convergence accuracy), we modified the ACO with AFSA and then developed a new optimization algorithm named AFSA-ACO. The details are introduced as follows.

\subsubsection{ACO}

ACO is a heuristics optimization algorithm, proposed by Dorigo, that mimics the cooperative behavior of ant colonies and is has been used to handle the numerous combinatorial optimization problems. It has been proven that the ACO algorithm has robust ability to search near-optimum solutions [26]. When ants search for foods, each ant will try its best to find the optimum routes between the nest and food source. In this process, the ants leave a chemical trail of pheromones on the ground, which guides the behavior of other ants. However, as time passes, the pheromones evaporate. Therefore, the pheromones must be enhanced through more 
ants, so the pheromones will not disappear. The main steps of ACO can be summarized as follows:

$\underline{\text { Step1. Initialization }}$

Generate $S_{0}$ initial solution

Step2. Local search

Use the local search procedure to solution $S_{0}$.

Repeat

$\underline{\text { Step3. Perturbation }}$

Perturb solution $S_{0}$ and obtain new solution $S^{\prime}$.

Step4. Local search

Use the local search procedure to solution S' and obtain solution S'.

Step5. Acceptance criterion

Perform acceptance criterion

Until termination condition met

\subsubsection{AFSA}

In the water, fish can find nutrients alone or follow other fish to find more nutrients. Thus, the highest number of local fish that survive generally is the highest concentration of nutrients in the water. The Artificial fish algorithm is based on this characteristic and constructs artificial fish to simulate fish feed, cluster and collision behavior and thereby realize optimization.

(1) Preying behavior

Assuming the present situation of an artificial fish is $X_{i}$, then randomly choose a new state $X_{j}$. It is well known that we can convert a maximum problem into the minimum problem. Hence in this case, we just take the maximum problem as an example in the following discussions. The selected fish should move a step in that direction if the maximum problem $Y_{i}<Y_{j}$; otherwise, select a state, $X_{j}$, randomly one time and determine whether it meets the pre-set forward situation. If it cannot meet the situation, it will move one step at random. The rules of step moving can be expressed by

$$
\begin{cases}X_{i+1}=X_{i}+\text { Step } \frac{X_{j}-X_{i}}{\left\|X_{j}-X_{i}\right\|} & \left(Y_{j}>Y_{i}\right) \\ X_{i+1}=X_{i}+\text { Step } & \left(Y_{j} \leq Y_{i}\right)\end{cases}
$$

(2) Clustering behavior

Artificial fish at present situation $X_{i}$ search for the partners' number NF and the central position $X,\left(d_{i j}<\right.$ Visual $)$; if $Y_{c} / N F>\delta Y_{i}$, it represents that enough food is at the center of the fish colony, where it is not too crowded. The related mathematical simulation formulas of the swarming behavior can be expressed as follows:

$$
\left\{\begin{array}{cc}
X_{i+1}=X_{i}+\text { Step } \frac{X_{c}-X_{i}}{\left\|X_{c}-X_{i}\right\|} & \left(Y_{c} / N F>\delta Y_{i} \text { and } N F \geq 1\right) \\
X_{i+1}=\text { Defalut Formula } & \left(Y_{c} / N F \leq \delta Y_{i} \text { or } N F=0\right)
\end{array}\right.
$$

(3) Following behavior

Assume that $X_{i}$ is the present state of AF looking for partner $X_{\max }$ near $Y_{\max }$, if $Y_{\max } / N F>\delta Y_{i}$, we can see that the present position of partner $X_{\max }$ possesses higher 
food consistence and it can be not crowded. The AF will take a step toward partner $X_{\max }$; otherwise, to the process of the searching behavior will be run.

The behavior can be expressed by the following mathematic description:

$$
\left\{\begin{array}{cc}
X_{i+1}=X_{i}+\text { Step } \frac{X_{c}-X_{i}}{\left\|X_{c}-X_{i}\right\|} & \left(Y_{\max } / N F>\delta Y_{i} \text { and } N F \geq 1\right) \\
X_{i+1}=\text { Defalut Formula } & \left(Y_{\max } / N F \leq \delta Y_{i} \text { or } N F=0\right)
\end{array}\right.
$$

(4) Behavior selection

Based on the problem which should be addressed, we can choose a behavior to simulate after evaluating the current environment of the AFSA. Trial approaches have been continually taken to simulate fish behaviors, and the best solutions can be implemented after evaluation. Here, we call the three biological behaviors of fish swarm as the searching behavior, the following behavior and the clustering behavior.

(5) Bulletin

Bulletin can be utilized to memorize the AF's best situation and the optimal solutions of the given problem. Every AF updates and compares its own state with the bulletin after moving. If the value on the bulletin is worse than the present state of the AF, the bulletin will be replaced. The pseudocode of the AFSA algorithm is as follows:

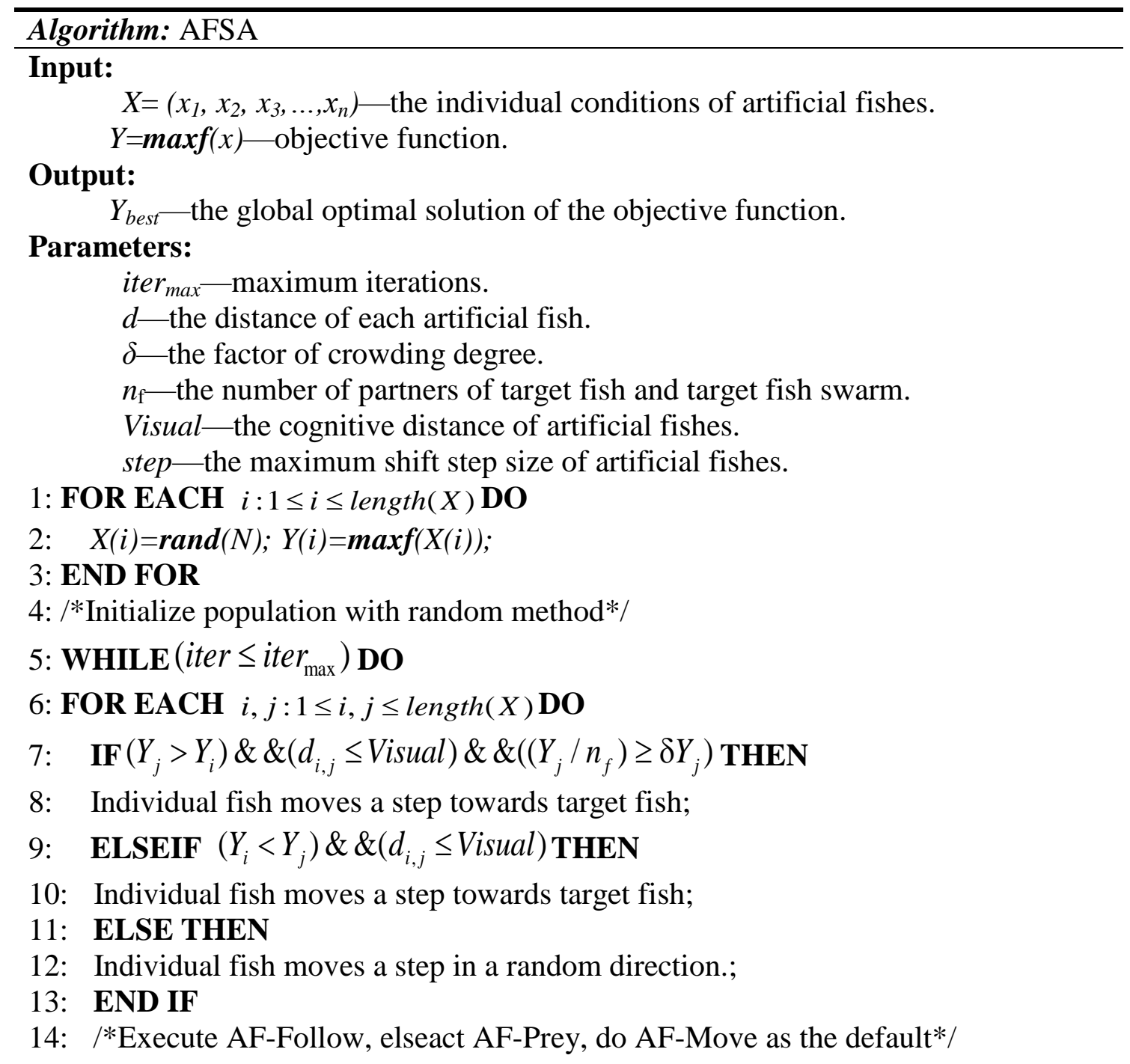


15: $\quad$ IF $\left(Y c>Y_{i}\right) \& \&\left(d_{i, j} \leq\right.$ Visual $) \& \&\left(\left(Y c / n_{f}\right) \geq \delta Y_{j}\right)$ THEN

16: Individual fish moves random steps towards target fish swarm;

17: ELSEIF $\left(Y_{i}<Y_{j}\right) \& \&\left(d_{i, j} \leq\right.$ Visual $)$ THEN

18: Individual fish moves a step towards target fish;

19: ELSE THEN

20: Individual fish moves a step in a random direction;

21: END IF

22: /*Execute AF-Swarm, elseact AF-Prey, do AF-Move as the default*/

23: Choose the better result of AF-Swarm and AF-Fellow by maxf;

24: Test whether the result is eligibility, break the circulation if the result is eligibility;

25: iter=iter+1;

26: END WHILE

3.2.3 AFSA-ACO

ACO possesses strong global exploration and exploitation capabilities. Moreover, the convergence speed of this algorithm is fast in early optimization [27]. Nevertheless, in the later optimization process, its convergence speed and accuracy decrease. To address these shortcomings, a novel modified algorithm on the basis of the AFSA algorithm, named AFSA-ACO, is proposed in this study. A rudimentary AFSA-ACO

351 algorithm is shown as follows:

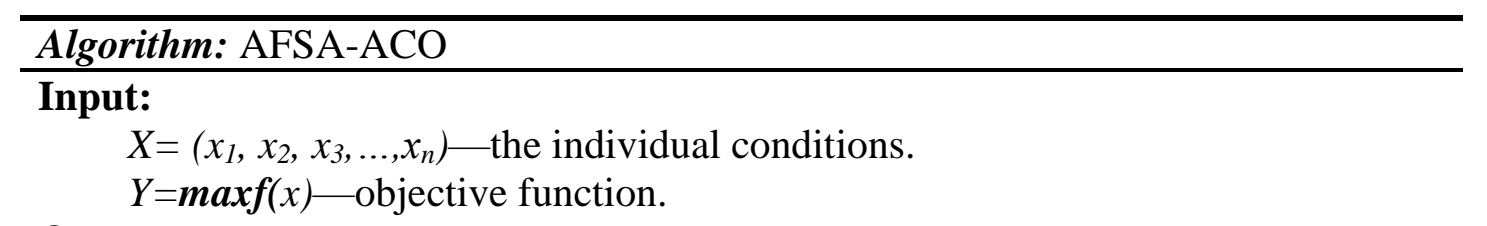

$X=\left(x_{1}, x_{2}, x_{3}, \ldots, x_{n}\right)$ - the individual conditions

Output:

Ybest - the global optimal solution of the objective function.

Parameters:

$g_{\text {ratio }}$ - minimum update rate for AFSA.

$\mathrm{N}$-maximum iterations of ACO.

iter $_{\text {max }}$ - maximum iterations of AFSA.

$\tau$ - the concentration of pheromone.

$\rho$ - pheromone volatilization coefficients.

$\eta$-inspiring factor.

1: Initialize initial parameter and generate initial fish school;

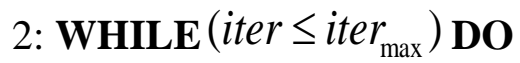

3: Use AFSA to get the optimal solution;

4: IF $\left(g_{\text {end }}<g_{\text {ratio }}\right)$ THEN

5: Break the iteration of AFSA, export the result Ybest $_{A F S A}$;

6: END IF

7: END WHILE

8: /*Use AFSA to get the fast-optimal solution for the initial ant colony*/

9: Initialize initial pheromone by $\tau_{\max }=\frac{1}{2(1-\rho)} \cdot \frac{1}{L_{b e s t}}$ and the results of AFSA;

10: WHILE $(1 \leq i, j \leq N)$ DO 
11: $p_{i j}^{k}(t)=\frac{\left[\tau_{i j}(t)\right]^{\alpha} \cdot\left[\eta_{i j}(t)\right]^{\beta}}{\sum_{h \in U_{i}^{k}}\left[\tau_{i j}(t)\right]^{\alpha} \cdot\left[\eta_{i j}(t)\right]^{\beta}}$

12: /* Calculate the transition probability*/

13: $\tau_{i j}(t+1)=(1-\rho) \tau_{i j}(t)+\Delta \tau_{i j}(t) ; \Delta \tau_{i j}(t)=\rho \times f\left(L_{b e s t}\right)$

14: /*Updating the pheromone and $L_{b e s t}$ is the optimal solution at present*/ 15: END WHILE

3.2.4 Test functions for validation of ACO and AFSA-ACO

In this section, four test functions, named Sphere, Rosenbrock, Rastrigin and Schaffer, as shown in Table 1, are employed to test the optimization performance of ACO and the developed optimized algorithm AFSA-ACO. The test of ACO and AFSA-ACO was performed in MATLAB R2012b on Windows 8 with a $1.80 \mathrm{GHz}$ Intel Core i7 4500U, 64 bits and 8 GB RAM. The ACO and AFSA-ACO parameters are given in Table 2. These experimental parameters are widely accepted for solving optimization problems and have usually obtained good results [26].

Table 1 Test functions for validation of ACO and AFSA-ACO

\begin{tabular}{|c|c|c|c|}
\hline $\begin{array}{c}\text { Function } \\
\text { name }\end{array}$ & Test function & Variable domain & Global optimum \\
\hline Sphere & $f(x)=\sum_{i=1}^{d} x_{i}^{2}$ & $x_{i} \in[-5.12,5.12]$ & $f_{\min }(0,0,0 \cdots 0)=0$ \\
\hline Rosenbrock & $f(X)=\sum_{i=1}^{d-1}\left[100\left(x_{i}^{2}-x_{i+1}\right)^{2}+\left(x_{i}-1\right)^{2}\right.$ & $x_{i} \in[-2.084,2.084]$ & $f_{\min }(1,1,1 \cdots 1)=0$ \\
\hline Rastrigin & $f(X)=\sum_{i=1}^{d}\left(x_{i}^{2}-10\left(2 \pi x_{i}\right)+10\right)$ & $x_{i} \in[-5.12,5.12]$ & $f_{\min }(0,0,0 \cdots 0)=0$ \\
\hline Schaffer & $f(X)=\frac{\sqrt{i=1}}{\left[1+0.001\left(\sum_{i=1}^{d} x_{i}^{2}\right)\right]^{2}}+0.5$ & $x_{i} \in[-5.12,5.12]$ & $f_{\min }(0,0,0 \cdots 0)=0$ \\
\hline
\end{tabular}

Table. 2 The experimental parameters of ACO and AFSA-ACO

\begin{tabular}{ccc}
\hline Experimental parameters & ACO & AFSA-ACO \\
\hline Maximum generation & 10000 & 10000 \\
Population size & 100 & 100 \\
A & 0.1 & 0.1 \\
$\beta_{0}$ & $1.0 / 3.5$ & $1.0 / 3.5$ \\
$\Gamma$ & 0.001 & 0.001 \\
Convergence tolerance & $10^{-5}$ & $10^{-5}$ \\
\hline
\end{tabular}

Based on the experimental results (as shown in Table 3), we present several conclusions, summarized as follows: 
- For the Sphere function, when the dimension is 10, the maximum, minimum and average values of the iteration of ACO and AFSA-ACO are 175 and 16, 154 and 3, and 162 and 7.4, respectively. When the dimension is 20, the maximum, minimum and average values of the iteration of ACO and AFSA-ACO are 242 and 42, 134 and 19 , and 175 and 28.6, respectively. When the dimension is 50, the values of the iteration of ACO and AFSA-ACO are 477 and 150, 365 and 72, and 397 and 101.3, respectively.

- For the Rosenbrock function, When the dimension is 2, the maximum, minimum and average values of the iterations of AFSA-ACO are 142, 63 and 92, respectively. ACO cannot obtain optimized results.

- Based on the analysis, similar results occur to Rastrigin and Chaffer functions, which also means that the performance of the AFSA-ACO algorithm is better than that of the ACO method. Details are shown in Table 3.

\begin{tabular}{|c|c|c|c|c|c|c|}
\hline Test function & Dimension & Algorithm & $\begin{array}{c}\text { Max } \\
\text { value of } \\
\text { iteration }\end{array}$ & $\begin{array}{c}\text { Min } \\
\text { value of } \\
\text { iteration }\end{array}$ & $\begin{array}{l}\text { Average } \\
\text { value of } \\
\text { iteration }\end{array}$ & $\begin{array}{c}\text { Convergence } \\
\text { rate }\end{array}$ \\
\hline \multirow{6}{*}{ Sphere } & \multirow{2}{*}{10} & $\mathrm{ACO}$ & 175 & 154 & 162 & 1 \\
\hline & & AFSA-ACO & 16 & 3 & 7.4 & 1 \\
\hline & \multirow{2}{*}{20} & ACO & 252 & 134 & 175 & 1 \\
\hline & & AFSA-ACO & 42 & 19 & 28.6 & 1 \\
\hline & \multirow{2}{*}{50} & ACO & 477 & 365 & 397 & 1 \\
\hline & & AFSA-ACO & 150 & 72 & 101.3 & 1 \\
\hline \multirow{2}{*}{ Rosenbrock } & \multirow{2}{*}{2} & $\mathrm{ACO}$ & - & - & - & - \\
\hline & & AFSA-ACO & 142 & 63 & 92 & 1 \\
\hline \multirow{6}{*}{ Rastrigin } & \multirow{2}{*}{10} & ACO & 397 & 303 & 351 & 1 \\
\hline & & AFSA-ACO & 172 & 119 & 148 & 1 \\
\hline & \multirow{2}{*}{20} & ACO & 742 & 651 & 683 & 0.84 \\
\hline & & AFSA-ACO & 477 & 391 & 435 & 1 \\
\hline & \multirow{2}{*}{50} & ACO & - & - & - & - \\
\hline & & AFSA-ACO & 1246 & 847 & 1037 & 0.87 \\
\hline \multirow{2}{*}{ Schaffer } & \multirow{2}{*}{2} & $\mathrm{ACO}$ & 1105 & 891 & 965 & 0.77 \\
\hline & & AFSA-ACO & 71 & 27 & 52 & 1 \\
\hline
\end{tabular}

Remark: Using the above experiments, we compare the maximum, minimum 379 and average iteration values of these two optimization algorithms. The developed 380 AFSA-ACO algorithm performed better under all condition. Therefore, the 381 developed AFSA-ACO algorithm was used to construct the developed combined 382 model.

383 3.2.5 Experiment: Test the performance of ACO and AFSA-ACO

384 To evaluate the exploration and exploitation capability of the AFSA-ACO, a 385 metric named excellent rate $\left(E R_{\text {index }}\right)$ is defined in this paper, which can provide the 386 performance information of the optimization algorithm:

$$
E R_{\text {index }}=\frac{n_{\text {better }}}{N_{\text {test }}} \times 100 \%
$$


where $n_{\text {better }}$ denotes the number that AFSA-ACO has better results than ACO. $N_{\text {test }}$ denotes the total experiment times.

In the experiment, several cases from original wind speed series are random selected to conduct the comparison test between the ACO and AFSA-ACO, and each case was experimented 100 times. The typical results are shown as Table 4. As shown in Table 4, the $E R_{\text {index }}$ of AFSA-ACO are commonly as $90 \%-95 \%$ and the best result can reach to $97 \%$. Thus, the developed optimization algorithm AFSA-ACO can

\begin{tabular}{lcccccccccc}
\hline Case & case 1 & case 2 & case 3 & case 4 & case 5 & case 6 & case 7 & case 8 & case 9 & case 10 \\
$E R_{\text {index }}$ & $92 \%$ & $90 \%$ & $89 \%$ & $92 \%$ & $97 \%$ & $88 \%$ & $94 \%$ & $90 \%$ & $92 \%$ & $93 \%$ \\
\hline
\end{tabular}

\section{9}

400

401

402

403

404

405

\section{Results and analysis}

To avoid accidental errors and increase the reliability of the models, each experiment was repeated twelve times and the average value was considered as the forecasting performance of one model. All of the simulations operated in the following computing environment as MATLAB R2012b on Windows 8 with a 1.80 GHz(4CPUs) Intel Core i7 4500U, 64 bits and 8 GB RAM.

\subsection{Data analysis and preprocessing}

The wind speed data indicate a characteristic of indeterminacy. Based on detailed analysis, several characteristics can be summarized:

- An alteration of wind speed led to stronger randomness, with the annual wind speed time series, which showed no significant trend.

- In the wind speed time series, both linear and nonlinear data characteristic appeared; the volatility, intermittency and randomness were all observed.

- The wind speed data presented different change features between morning and night. An obvious variation trend can be observed in the data at night, and the diurnal data are different.

\section{One-Step Ten-Minute}

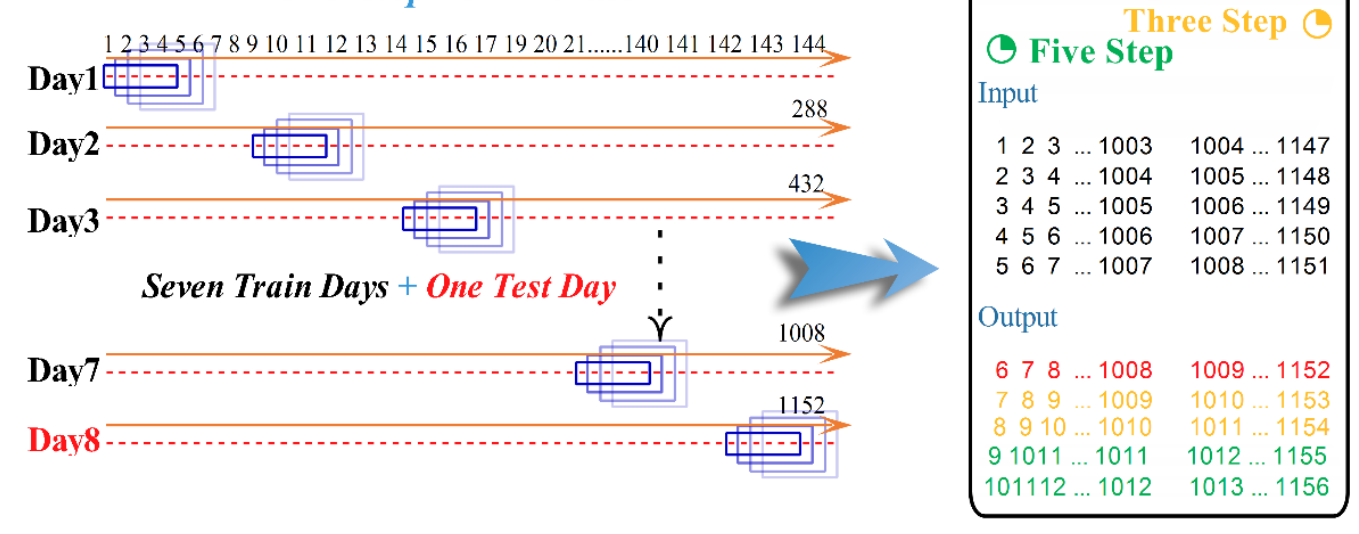

Fig. 3 The process of longitudinal data selection 
For this case studies, the data were randomly selected from the ten-minute wind speed data from 2011 in Shandong, China. Fig. 3 shows the process of the longitudinal data selection procedure for the ten-minute wind speed data as well as the input/output dataset for one-step and multi-step forecasting. In addition, Fig. 4 provides a brief description of the experiment data used in this paper. For the experiments, first, the wind speed data were divided into several series: a data series included eight days, and some of series were randomly selected to conduct the experiment (the experiment were based on both the linear and nonlinear model). Second, the final results are the average values of these experiments and were divided into the diurnal dataset and nightly dataset for simulation and analysis.

Moreover, to develop an optimized wind speed forecasting model and achieve a better forecasting performance, considering the characteristics of the original wind speed data, some data preprocessing technique that have successfully used in several hybrids forecasting model have been used in the first stage to reduce the negative influence of the feature of random disturbance in the original data. In this paper, the singular spectrum analysis (SSA) is introduced as a decomposing and de-nosing technique to decompose the original data into filtered data in the data preprocessing stage. The details of SSA can be seen in [19].

\subsection{The performance metric}

Many performance metrics have been employed to verify the forecasting ability of different models. However, there is no universal standard for error evaluation [28]. Therefore, as generally recognized criteria, three evaluation standards were adopted to show and compare the forecasting effectiveness of the models, as shown in Table 5. 


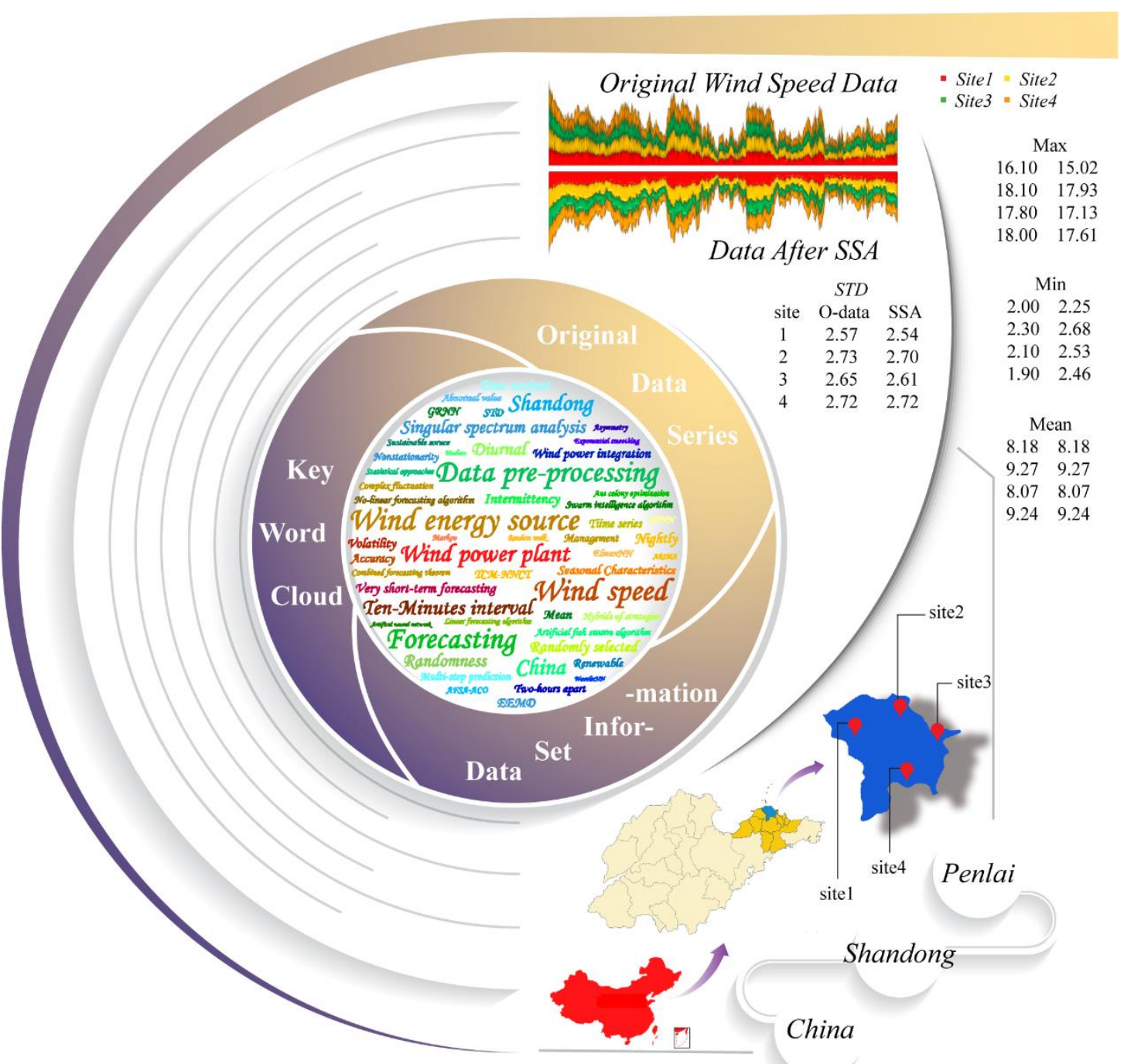

441

Fig. 4 A brief description of the data used in this paper

Table 5 Three metric rules for model performance

\begin{tabular}{ccc}
\hline Standard & Definition & Equation \\
MAE & $\begin{array}{c}\text { The mean absolute error of } n \text { times } \\
\text { prediction results }\end{array}$ & MAE $=\frac{1}{N} \sum_{i=1}^{N}\left|y_{i}-\hat{y_{i}}\right|$ \\
MSE & $\begin{array}{c}\text { The mean square error of } n \text { times } \\
\text { prediction results }\end{array}$ & MSE $=\frac{1}{N} \sum_{i=1}^{N}\left(y_{i}-\hat{y}_{i}\right)^{2}$ \\
MAPE & $\begin{array}{c}\text { The mean absolute percentage } \\
\text { error of } n \text { times prediction results }\end{array}$ & MAPE $=\frac{1}{N} \sum_{i=1}^{N}\left|\frac{y_{i}-\hat{y}_{i}}{y_{i}}\right| \times 100 \%$
\end{tabular}
length of testing data. The MAE shows the level of similarity between the forecasting The MAPE is a unit-free standard and is sensitive to small changes in the results. Moreover, a larger value of the above metric indicates worse accuracy. 


\subsection{The experiment with nonlinear algorithm}

To capture the nonlinear characteristic of the wind speed data, four artificial neutral networks models, i.e., BPNN, GRNN, ENN and WNN, are proposed for wind speed forecasting in this section.

\subsubsection{The forecasting results of the BPNN model}

To obtain the number of artificial neurons, the trial and error method [29] is used in this paper. The experiment parameters of BPNN model are selected through many experiments, which are shown in Table 6.

Table 6 The experiment parameters of BPNN

\begin{tabular}{cc}
\hline Experimental parameters & Default value \\
\hline The number of input layer neurons & 5 \\
The number of hidden layer neurons & $1-11$ \\
The number of output layer neurons & $1 ; 3 ; 5$ \\
The training velocity & 0.1 \\
The maximum number of iteration & 1000 \\
Object accuracy & 0.00004 \\
\hline
\end{tabular}

The typical forecasting results of BPNN model are given in Table 7. Several conclusions can be summarized as follows:

- For different time periods in one day, the BPNN model presents different forecasting accuracies, which indicates that wind speed has the feature of volatility, intermittent and instability. Taking one-step forecasting as an example, at site 1 , the minimum MAPE value for one day is $1.99 \%$ at 22:00-23:50, its maximum is $7.06 \%$ at 10:00-11:50, and its daily average the is $4.64 \%$.

- For different sites, the BPNN model shows a relatively smaller difference in terms of forecasting performance, which is within the acceptable range. For example, the average MAPE values of the BPNN model for one-step forecasting are $4.64 \%, 4.55 \%, 4.87 \%$ and $3.87 \%$ corresponding to the MAPE values for three-step forecasting of $5.88 \%, 5.30 \%, 6.08 \%$ and $5.51 \%$ from site 1 to site 4 , respectively.

- Based on the daily average forecasting accuracy in different sites, the averaged value of MAPE can be considered the performance of one model. Therefore, the MAPE value are $4.48 \%, 5.69 \%$ and $8.78 \%$ for one, three and five-step forecasting, respectively.

Remark: Although the average forecasting accuracy is relative better, the BPNN model presents different forecasting performances in different time periods. Therefore, we can conclude that the BPNN model is unstable for wind speed forecasting. 
Table 7 Typical results of the BPNN model for multi-step wind speed forecasting

\begin{tabular}{|c|c|c|c|c|c|c|c|c|c|c|c|c|c|c|c|}
\hline \multicolumn{3}{|c|}{ Time node } & \multirow{2}{*}{$\begin{array}{l}0: 00 \\
1: 50\end{array}$} & \multirow{2}{*}{$\begin{array}{l}2: 00 \\
3: 50\end{array}$} & \multirow{2}{*}{$\begin{array}{l}4: 00 \\
5: 50\end{array}$} & \multirow{2}{*}{$\begin{array}{l}6: 00 \\
7: 50\end{array}$} & \multirow{2}{*}{$\begin{array}{l}8: 00 \\
9: 50\end{array}$} & \multirow{2}{*}{$\begin{array}{l}10: 00 \\
11: 50\end{array}$} & \multirow{2}{*}{$\begin{array}{l}12: 00 \\
13: 50\end{array}$} & \multirow{2}{*}{$\begin{array}{l}14: 00 \\
15: 50\end{array}$} & \multirow{2}{*}{$\begin{array}{l}16: 00 \\
17: 50\end{array}$} & \multirow{2}{*}{$\begin{array}{l}18: 00 \\
19: 50\end{array}$} & \multirow{2}{*}{$\begin{array}{l}20: 00 \\
21: 50\end{array}$} & \multirow{2}{*}{$\begin{array}{l}22: 00 \\
23: 50\end{array}$} & \multirow{2}{*}{$\begin{array}{c}\text { Daily } \\
\text { average }\end{array}$} \\
\hline Step & Metric & Site & & & & & & & & & & & & & \\
\hline \multirow{12}{*}{ One-step ahead } & \multirow{4}{*}{ MAE } & site1 & 0.2895 & 0.3413 & 0.1993 & 0.2115 & 0.2972 & 0.3712 & 0.2847 & 0.3503 & 0.2408 & 0.1475 & 0.3007 & 0.2218 & 0.2713 \\
\hline & & site2 & 0.2841 & 0.2516 & 0.206 & 0.2055 & 0.3671 & 0.3322 & 0.3346 & 0.3235 & 0.3425 & 0.2472 & 0.4895 & 0.3034 & 0.3073 \\
\hline & & site3 & 0.1353 & 0.1715 & 0.1707 & 0.2235 & 0.2879 & 0.3919 & 0.221 & 0.3272 & 0.3086 & 0.1831 & 0.5613 & 0.2962 & 0.2732 \\
\hline & & site 4 & 0.2088 & 0.1362 & 0.1608 & 0.1314 & 0.1881 & 0.3568 & 0.2256 & 0.2564 & 0.3614 & $0.24 \mathrm{~s} 91$ & 0.4215 & 0.5211 & 0.2681 \\
\hline & \multirow{4}{*}{ MAPE } & site1 & 6.51 & 6.3 & 2.81 & 2.81 & 5.84 & 7.06 & 6.56 & 6.65 & 3.44 & 2.23 & 3.49 & 1.99 & 4.64 \\
\hline & & site2 & 5.41 & 4.6 & 3.01 & 2.56 & 6.41 & 6.26 & 6.14 & 5.13 & 4.37 & 2.94 & 5.31 & 2.43 & 4.55 \\
\hline & & site3 & 2.39 & 4.06 & 3.35 & 3.51 & 5.5 & 9.68 & 5.42 & 6.81 & 5.31 & 2.96 & 6.95 & 2.49 & 4.87 \\
\hline & & site 4 & 4.45 & 2.9 & 2.44 & 1.73 & 3.51 & 7.64 & 3.86 & 3.98 & 4.29 & 2.89 & 4.42 & 4.27 & 3.87 \\
\hline & \multirow{4}{*}{ MSE } & site1 & 0.1035 & 0.1989 & 0.0626 & 0.0579 & 0.1608 & 0.2732 & 0.1159 & 0.1852 & 0.0999 & 0.0317 & 0.1424 & 0.0709 & 0.1252 \\
\hline & & site2 & 0.1042 & 0.0779 & 0.0703 & 0.0677 & 0.1907 & 0.1638 & 0.1365 & 0.1633 & 0.2057 & 0.0788 & 0.294 & 0.1399 & 0.1411 \\
\hline & & site3 & 0.0295 & 0.0465 & 0.0478 & 0.0914 & 0.1131 & 0.2345 & 0.0741 & 0.1752 & 0.1494 & 0.0502 & 0.3819 & 0.1132 & 0.1256 \\
\hline & & site 4 & 0.0679 & 0.0292 & 0.0437 & 0.0232 & 0.066 & 0.1836 & 0.0762 & 0.0995 & 0.1783 & 0.0972 & 0.2159 & 0.3806 & 0.1218 \\
\hline \multirow{12}{*}{ Three-step ahead } & \multirow{4}{*}{ MAE } & site1 & 0.3809 & 0.3341 & 0.3053 & 0.1974 & 0.4523 & 0.3904 & 0.3776 & 0.4909 & 0.3162 & 0.1812 & 0.5198 & 0.3158 & 0.3552 \\
\hline & & site2 & 0.3065 & 0.2767 & 0.2753 & 0.3234 & 0.4251 & 0.3473 & 0.4346 & 0.3784 & 0.3621 & 0.3074 & 0.6098 & 0.3869 & 0.3695 \\
\hline & & site3 & 0.2041 & 0.2238 & 0.2812 & 0.2801 & 0.4297 & 0.4199 & 0.2657 & 0.4078 & 0.3035 & 0.2697 & 0.6514 & 0.3682 & 0.3421 \\
\hline & & site 4 & 0.2752 & 0.2342 & 0.3222 & 0.2134 & 0.3115 & 0.455 & 0.4968 & 0.296 & 0.4084 & 0.316 & 0.5948 & 0.5501 & 0.3728 \\
\hline & \multirow{4}{*}{ MAPE } & site1 & 7.56 & 6.35 & 4.31 & 2.62 & 8.87 & 7.56 & 8.01 & 9.41 & 4.52 & 2.8 & 5.78 & 2.81 & 5.88 \\
\hline & & site2 & 5.69 & 4.99 & 3.82 & 4.17 & 7.44 & 6.47 & 7.89 & 5.79 & 4.46 & 3.79 & 6.02 & 3.1 & 5.3 \\
\hline & & site3 & 3.63 & 5.64 & 5.62 & 4.33 & 8.76 & 10.15 & 6.54 & 8.24 & 5.2 & 4.48 & 7.25 & 3.1 & 6.08 \\
\hline & & site 4 & 5.9 & 5.11 & 5 & 2.89 & 5.92 & 9.53 & 8.5 & 4.52 & 4.66 & 3.79 & 5.83 & 4.52 & 5.51 \\
\hline & \multirow{4}{*}{ MSE } & site1 & 0.2776 & 0.1699 & 0.1327 & 0.0606 & 0.3764 & 0.2787 & 0.2275 & 0.3815 & 0.1572 & 0.0536 & 0.5783 & 0.1538 & 0.2373 \\
\hline & & site2 & 0.1292 & 0.1065 & 0.1488 & 0.1684 & 0.2799 & 0.1846 & 0.2779 & 0.2211 & 0.2021 & 0.1362 & 0.6202 & 0.2608 & 0.228 \\
\hline & & site3 & 0.0747 & 0.0876 & 0.1261 & 0.1589 & 0.2963 & 0.2739 & 0.1151 & 0.2617 & 0.1535 & 0.1026 & 0.7028 & 0.1744 & 0.2106 \\
\hline & & site 4 & 0.134 & 0.104 & 0.1822 & 0.0838 & 0.1721 & 0.2962 & 0.4328 & 0.1491 & 0.2158 & 0.1585 & 0.6475 & 0.4545 & 0.2526 \\
\hline \multirow{11}{*}{ Five-step ahead } & & site1 & 0.4723 & 0.3806 & 0.4581 & 0.3309 & 0.6648 & 0.4168 & 0.6778 & 0.6689 & 0.416 & 0.2899 & 1.1385 & 0.5154 & 0.5358 \\
\hline & $\mathrm{MAF}$ & site2 & 0.3741 & 0.4061 & 0.5466 & 0.5572 & 0.6009 & 0.4022 & 0.8178 & 0.5323 & 0.5608 & 0.4083 & 0.9742 & 0.6084 & 0.5657 \\
\hline & MIAE & site3 & 0.3931 & 0.417 & 0.5527 & 0.3831 & 0.7186 & 0.5507 & 0.4672 & 0.5683 & 0.3911 & 0.3924 & 1.086 & 0.5334 & 0.5378 \\
\hline & & site4 & 0.4388 & 0.4694 & 0.5452 & 0.3527 & 0.6237 & 0.6274 & 0.9474 & 0.4717 & 0.5739 & 0.5226 & 1.0543 & 0.7151 & 0.6118 \\
\hline & & site1 & 9.04 & 7.09 & 6.39 & 4.6 & 13.26 & 8.59 & 13.8 & 12.74 & 5.9 & 4.55 & 11.63 & 4.53 & 8.51 \\
\hline & $\mathrm{MAPF}$ & site2 & 6.84 & 7.13 & 7.13 & 7.66 & 10.83 & 7.56 & 14.74 & 7.88 & 6.68 & 5.24 & 9.06 & 4.81 & 7.96 \\
\hline & MAPE & site3 & 6.9 & 11.17 & 10.31 & 6.12 & 15.68 & 13.02 & 11.59 & 10.88 & 6.8 & 6.73 & 10.97 & 4.44 & 9.55 \\
\hline & & site 4 & 9.31 & 9.79 & 8.18 & 4.94 & 11.95 & 13.25 & 16.43 & 6.88 & 6.31 & 6.57 & 9.77 & 5.8 & 9.1 \\
\hline & & site1 & 0.4785 & 0.2318 & 0.385 & 0.1906 & 0.7594 & 0.302 & 0.9534 & 0.7514 & 0.2685 & 0.1613 & 2.7962 & 0.4543 & 0.6444 \\
\hline & MSE & site2 & 0.2166 & 0.2832 & 0.553 & 0.6276 & 0.6418 & 0.2648 & 1.0753 & 0.485 & 0.4905 & 0.281 & 1.9305 & 0.7473 & 0.633 \\
\hline & & site3 & 0.2993 & 0.3574 & 0.5667 & 0.2737 & 0.9434 & 0.4791 & 0.3913 & 0.5311 & 0.2961 & 0.2359 & 2.6131 & 0.4638 & 0.6209 \\
\hline
\end{tabular}




\subsubsection{The forecasting results of the GRNN model}

The experiment parameters of the GRNN model are shown in Table 8. Moreover, the radial basis function expansion range is $[0.1,2.0]$, which is performed to establish the superior GRNN model. The one-step and multi-step forecasting results in diurnal and nightly, are shown in Table 9. For one-step forecasting, the GRNN model has a minimum MAPE value of $6.42 \%$ in the nightly wind speed, compared to the MAPE of $8.62 \%$ for the diurnal speed; we can reach the same conclusion for the multi-step forecasting.

Remark: The GRNN model performs better than the BPNN model in terms of forecasting stability, but it is worse than the BPNN model regarding accuracy. Moreover, the forecasting accuracy of the GRNN model for the nightly wind speed forecasting is better than the diurnal, which may account for the diurnal wind speed change being more drastic than the nightly change.

Table 8 Experimental parameters of GRNN

\begin{tabular}{cc}
\hline Experimental parameters & Default value \\
\hline iteration time & 100 \\
learning rate & 0.1 \\
training requirement accuracy & 0.00004 \\
\hline
\end{tabular}

Table 9 Forecasting results of GRNN

\begin{tabular}{l|llll|lllcc}
\hline \multicolumn{2}{c}{ Diurnal } & MAE & MAPE & \multicolumn{1}{c}{ MSE } & \multicolumn{2}{c}{ Nightly } & MAE & MAPE & MSE \\
\hline \multirow{3}{*}{ One } & Site1 & 0.5014 & 9.58 & 0.4125 & & Site1 & 0.5033 & 7.04 & 0.4620 \\
-step & Site2 & 0.5259 & 8.62 & 0.4163 & One & Site2 & 0.5157 & 6.42 & 0.5021 \\
& Site3 & 0.4935 & 10.46 & 0.4120 & -step & Site3 & 0.5424 & 8.25 & 0.6330 \\
& Site4 & 0.5943 & 9.88 & 0.4903 & & Site4 & 0.5695 & 8.16 & 0.6221 \\
Three & Site1 & 0.6385 & 12.22 & 0.6941 & & Site1 & 0.6811 & 9.22 & 0.9228 \\
-step & Site2 & 0.6733 & 10.81 & 0.6857 & Three & Site2 & 0.7689 & 9.50 & 1.0908 \\
& Site3 & 0.6676 & 14.68 & 0.7487 & -step & Site3 & 0.8044 & 12.58 & 1.3454 \\
& Site4 & 0.7849 & 12.93 & 0.8520 & & Site4 & 0.7314 & 10.46 & 1.0706 \\
Five & Site1 & 0.7369 & 13.97 & 0.9373 & & Site1 & 0.8034 & 10.57 & 1.2923 \\
-step & Site2 & 0.8031 & 12.83 & 0.9494 & Five & Site2 & 0.9386 & 11.63 & 1.6297 \\
& Site3 & 0.7691 & 16.78 & 0.9395 & -step & Site3 & 0.9952 & 15.25 & 2.1607 \\
& Site4 & 0.9664 & 15.92 & 1.3716 & & Site4 & 0.9057 & 12.79 & 1.5458 \\
\hline
\end{tabular}

\subsubsection{The forecasting results of the ENN model}

In this case, the trial and error method are also used to obtain the best number of neurons. The best results are selected based on many experiments. The experimental parameters of ENN are presented in Table 10. To research the performance of the ENN model, the forecasting results (MAE, MAPE and MSE) of the ENN model for wind speed forecasting in diurnal and nightly speeds are shown in Table 11.

Remark: The experiment results reveals that the ENN model is also unstable and performs worse than GRNN and BPNN in terms of forecasting accuracy. Similar to

BPNN and GRNN, the forecasting accuracy of the ENN model for nightly wind speed

504 wind speed change is more drastic than the nightly change. 
Table 10 The experimental parameters of ENN

\begin{tabular}{cc}
\hline Experimental parameters & Default value \\
\hline The number of input layer neurons & 5 \\
The number of hidden layer neurons & $1-11$ \\
The number of output layer neurons & $1 ; 3 ; 5$ \\
iteration time & 100 \\
learning rate & 0.1 \\
training requirement accuracy & 0.00004 \\
\hline
\end{tabular}

Table 11 The typical results of ENN

\begin{tabular}{l|cccc|lllcc}
\hline \multicolumn{2}{c}{ Diurnal } & MAE & MAPE & \multicolumn{1}{l}{ MSE } & \multicolumn{2}{c}{ Nightly } & MAE & MAPE & MSE \\
\hline \multirow{4}{*}{ One } & Site1 & 0.4416 & 8.16 & 0.3225 & & Site1 & 0.4249 & 6.05 & 0.3306 \\
-step & Site2 & 0.4516 & 7.32 & 0.2962 & One & Site2 & 0.4614 & 5.77 & 0.3608 \\
& Site3 & 0.3884 & 8.00 & 0.2353 & -step & Site3 & 0.3717 & 5.79 & 0.2599 \\
& Site4 & 0.4342 & 7.18 & 0.2859 & & Site4 & 0.4443 & 6.41 & 0.3684 \\
& Site1 & 0.5534 & 10.43 & 0.5443 & & Site1 & 0.5313 & 7.32 & 0.6133 \\
Three & Site2 & 0.5775 & 9.27 & 0.4916 & Three & Site2 & 0.6571 & 7.92 & 0.8611 \\
-step & Site3 & 0.5189 & 11.12 & 0.4607 & - step & Site3 & 0.6494 & 10.37 & 0.9331 \\
& Site4 & 0.6071 & 9.77 & 0.5637 & & Site4 & 0.5799 & 7.99 & 0.7575 \\
& Site1 & 0.6898 & 12.93 & 0.8248 & & Site1 & 0.7156 & 9.38 & 1.1659 \\
Five & Site2 & 0.6736 & 10.65 & 0.7083 & Five & Site2 & 0.8255 & 9.89 & 1.4404 \\
-step & Site3 & 0.6811 & 14.69 & 0.7825 & -step & Site3 & 0.8536 & 13.28 & 1.6686 \\
& Site4 & 0.7701 & 12.39 & 0.9301 & & Site4 & 0.7329 & 9.87 & 1.2239 \\
\hline
\end{tabular}

$508 \quad$ 4.3.4 The forecasting results of $\mathrm{WNN}$

509 To establish the WNN model, the detailed parameters of WNN are set as shown

510 in Table 12. The typical results for one-step and multi-step forecasting at four sites 511 are presented in Table 13.

512 Remark: The forecasting stability of WNN is worse than the other three nonlinear 513 models. The forecasting performance are sometimes unacceptable and sometimes 514 acceptable in certain condition; however, even the best forecasting results are also 515 worse than those of the other neural networks.

Table 12 The experimental parameters of WNN

\begin{tabular}{cc}
\hline Experimental parameters & Default value \\
\hline iteration time & 100 \\
learning rate & 0.1 \\
training requirement accuracy & 0.00004 \\
\hline
\end{tabular}

Table 13 The typical results of WNN

\begin{tabular}{l|cccc|lcccc}
\hline \multicolumn{2}{c}{ Diurnal } & MAE & MAPE & \multicolumn{1}{c}{ MSE } & \multicolumn{2}{c}{ Nightly } & MAE & MAPE & MSE \\
\hline \multirow{3}{*}{ One- } & Site1 & 0.5900 & 12.16 & 0.5552 & & Site1 & 0.5178 & 8.68 & 0.4805 \\
step & Site2 & 1.0150 & 16.38 & 1.5297 & One & Site2 & 1.0531 & 12.72 & 2.0930 \\
& Site3 & 0.4853 & 10.40 & 0.3712 & -step & Site3 & 0.5138 & 8.37 & 0.5242 \\
Three & Site4 & 0.8425 & 13.66 & 1.0167 & & Site4 & 0.8717 & 12.27 & 1.4864 \\
- step & Site1 & 0.6638 & 12.56 & 0.7412 & Three & Site1 & 0.6509 & 8.83 & 0.8444 \\
& Site2 & 0.6565 & 10.44 & 0.6525 & -step & Site2 & 0.7671 & 9.35 & 1.1716
\end{tabular}




\begin{tabular}{|c|c|c|c|c|c|c|c|c|c|}
\hline \multirow{6}{*}{$\begin{array}{l}\text { Five } \\
\text {-step }\end{array}$} & Site3 & 0.8274 & 18.00 & 1.0716 & \multirow{6}{*}{$\begin{array}{l}\text { Five } \\
\text {-step }\end{array}$} & Site3 & 1.0144 & 15.98 & 2.0813 \\
\hline & Site4 & 0.7396 & 11.94 & 0.8109 & & Site4 & 0.7110 & 9.86 & 1.0524 \\
\hline & Site1 & 0.7222 & 13.60 & 0.8903 & & Site1 & 0.7644 & 10.06 & 1.2491 \\
\hline & Site2 & 0.7093 & 11.28 & 0.7711 & & Site2 & 0.8772 & 10.46 & 1.6118 \\
\hline & Site3 & 0.7299 & 15.67 & 0.8703 & & Site3 & 0.9132 & 14.15 & 1.8394 \\
\hline & Site4 & 0.8497 & 13.72 & 1.0879 & & Site4 & 0.8033 & 10.87 & 1.3907 \\
\hline
\end{tabular}

$518 \quad 4.4$ The experiment with the linear algorithm

519 To forecast the linear characteristic of the wind speed data, two linear algorithms, 520 including ARIMA and ES, are adopted as the linear section in the developed 521 combined forecasting model.

\section{4.4.1 The forecasting results of ARIMA}

523 To obtain the best parameter to adapt to ARIMA, the parameters $p, q$ and $r$ are 524 used with repeat trials from 1 to 15 , and the parameter $r$ is cycled from 1-4. The 525 typical results of ARIMA of the wind speed data at diurnal and nightly moments 526 using the one-step and multi-step are presented in Table 14. The maximum values of 527 diurnal MAPE and nightly MAPE are $4.24 \%$ and $3.36 \%$ for one step, and the 528 minimum value are $5.89 \%$ and $3.98 \%$, respectively.

529 4.4.2 The forecasting results of ES

530 To obtain the best parameter to adapt to ES, the parameter $\alpha$ is used with repeat 531 trials from 0.01 to 0.99 . The typical results of the ES of the wind speed data at diurnal 532 and nightly moments on multi-steps are presented in Table 14.

533 Remark: The forecasting stability of ARIMA is more stable than ES but worse 534 than BPNN and GRNN, and the forecasting performance is higher than that of ES and 535 ENN but is lower than BPNN and GRNN. 
Table 14 Typical results of ARIMA and ES for wind speed forecasting

\begin{tabular}{|c|c|c|c|c|c|c|c|c|c|c|c|c|c|}
\hline & & \multicolumn{4}{|c|}{ One step ahead } & \multicolumn{4}{|c|}{ Three-step ahead } & \multicolumn{4}{|c|}{ Five-step ahead } \\
\hline & MAE & 0.2834 & 0.2554 & 0.6046 & 0.5255 & 0.3936 & 0.3563 & 0.7282 & 0.7500 & 0.7459 & 0.7617 & 0.8319 & 0.9036 \\
\hline \multirow{2}{*}{ Site1 } & MSE & 0.1485 & 0.1001 & 0.6052 & 0.5622 & 0.2357 & 0.2289 & 0.9058 & 1.1791 & 0.8432 & 1.1202 & 1.1480 & 1.6564 \\
\hline & MAE & 0.3251 & 0.2912 & 0.6212 & 0.5901 & 0.3692 & 0.4010 & 0.7479 & 0.8770 & 0.7901 & 0.8675 & 0.8231 & 1.0894 \\
\hline \multirow[t]{2}{*}{ Site2 } & MAPE & 5.22 & 3.91 & 9.88 & 7.31 & 5.77 & 5.04 & 11.76 & 10.62 & 12.34 & 10.39 & 12.75 & 12.87 \\
\hline & MSE & 0.1585 & 0.1253 & 0.5821 & 0.7133 & 0.2090 & 0.2946 & 0.8550 & 1.5819 & 0.8962 & 1.3781 & 1.0827 & 2.3521 \\
\hline \multirow[t]{3}{*}{ Site3 } & MAPE & 5.89 & 3.73 & 12.66 & 8.55 & 8.03 & 6.48 & 16.30 & 14.19 & 16.63 & 14.77 & 17.64 & 18.50 \\
\hline & MSE & 0.1319 & 0.1140 & 0.6102 & 0.6742 & 0.2198 & 0.3511 & 0.9661 & 1.9252 & 0.8902 & 1.7768 & 1.0953 & 3.1395 \\
\hline & MAE & 0.2660 & 0.2610 & 0.5595 & 0.5713 & 0.4227 & 0.3779 & 0.8077 & 0.7800 & 0.8431 & 0.7559 & 0.9547 & 0.9285 \\
\hline \multirow[t]{2}{*}{ Site4 } & MAPE & 4.24 & 3.36 & 9.01 & 7.84 & 6.79 & 4.94 & 12.93 & 10.52 & 13.61 & 10.02 & 15.03 & 12.14 \\
\hline & MSE & 0.1124 & 0.1243 & 0.4905 & 0.6275 & 0.2568 & 0.2750 & 1.0123 & 1.2949 & 0.9775 & 1.1491 & 1.4453 & 1.8200 \\
\hline
\end{tabular}




\subsection{The forecasting results of the combined model and comparisons}

To prove the superiority of this combined model, some experiments were conducted Three performance metrics are used to conduct a comparative study with the individual models contained in the developed combined model were conducted, the bias-variance framework is employed to further evaluate the developed combined model.

\subsubsection{Experiment I: Testing the combined model with comparisons}

The experiment was performed to further show that the developed forecasting model integrated with the developed AFSA-ACO algorithm could improve the wind speed forecasting performance. The individual models, including two linear algorithms (ES and ARIMA) and four nonlinear algorithms (BPNN, ENN, WNN, GRNN), were employed as comparison models to conduct a comparative study and evaluate the developed combined model. The experiment was designed to perform one-step and multi-step wind speed forecasting, and each experiment and evaluate three times for each step case. The comparative study results are shown in Table 15 and Fig. 5., which shows the forecasting effectiveness for the experimental datasets by BPNN, ENN, GRNN, WNN, ARIMA, ES and the combined model in one, three and five-step forecasting in terms of three typical performance metrics: MAE, MAPE and MSE. According to the average value of the MAPE metric, whether for the one-step or multi-step forecasting, the developed combined model outperforms all benchmark models. For example, for one-step ahead forecasting, the MAPE values obtained by BPNN, ENN, GRNN, WNN, ARIMA, ES and the developed combined model are $4.71 \%, 7.16 \%, 6.34 \%, 9.44 \%, 5.25 \%, 10.25 \%$ and $4.48 \%$, respectively, while the MAPE value for five-step forecasting are $8.80 \%, 13.46 \%, 12.02 \%, 13.17 \%, 13.61 \%, 17.02 \%$ and $8.31 \%$, respectively. The BPNN model achieved second place, while the ES model was last for all horizon forecasting. With an increase in the forecasting horizon, the forecasting error became lager. Based on a comparison study of multi-step forecasting performance, the differences between one, three and five-step forecasting are $0.93 \%$ and $3.83 \%$, which is within the acceptable range. Thus, we conclude that the developed combined model can be successfully and effectively applied for multi-step forecasting

\subsubsection{Experiment II: Testing with bias-variance framework}

The bias-variance framework was employed to evaluate the forecasting accuracy and stability of one model, which is important for verifying the model's effectiveness. The absolute value of bias is considered the average difference between the actual value and the forecasting value, and the variance reveals the model's variability. A smaller absolute value of bias reveals a higher forecasting accuracy, while a smaller variance represents better stability. The comparison results of the bias-variance framework are shown in Table 16. As shown in Table 16, the absolute values of biases and variances are all smaller than other compared models, which proves that the developed model can achieve wind speed forecasting more accurately and provides more stability. Therefore, the developed combined forecasting model, with its higher accuracy and strong stability, is superior to the other compared models and can be an efficient technique in engineering applications.

4.5.3 Experiment III: Test the performance of the combined model through forecasting horizon.

To explore how the performance of the developed model decrease through the forecasting time horizon at the four examined sites, 1-tesp to 24-step ahead forecasting experiments were conducted in this section and the MAPE results are shown in Table 17. Two particular times (8:00 and 20:00) were chosen as the beginning of the prediction to 
conduct the experiment. In the next 4 hours forecasting horizon, the MAPE value of the Test-1 obtained from 1-step to 24-step are $4.50 \%, 8.80 \%, 14.79 \%, 20.33 \%$ and $30.87 \%$, respectively, and the MAPE value of Test-2 are 4.67\%, 9.13\%, 15.45\%, $18.46 \%$ and $31.79 \%$, respectively. By making a comparison from 1-step to 24-step, the greater the number of steps of the forecast, the lower the accuracy. The performance of the combined model form 1-step to 12-step can achieve relatively satisfied performance, however, when forecasting horizon beyond 12-step, the MAPE of the proposed model decreasing quickly. Thus, we can affirm that the developed combined model can be effectively applied for multi-step forecasting.

Table 17 The MAPE of combined model forecasted through the time horizon

\begin{tabular}{|c|c|c|c|c|c|c|}
\hline & forecast horizon & stie1 & site2 & site3 & site4 & mean \\
\hline \multirow{5}{*}{ Test-1 } & $8: 10(1-$ step $)$ & 4.57 & 4.32 & 4.82 & 4.29 & 4.50 \\
\hline & 9:00 (6-step) & 8.89 & 8.17 & 9.39 & 8.75 & 8.80 \\
\hline & 10:00 (12-step) & 14.42 & 14.94 & 15.62 & 14. 18 & 14.79 \\
\hline & 11:00 (18-step) & 20.97 & 21.08 & 20.33 & 18. 94 & 20.33 \\
\hline & 12:00 (24-step) & 31.14 & 30.56 & 32.41 & 29.37 & 30.87 \\
\hline \multirow{5}{*}{ Test-2 } & $20: 10$ (1-step) & 4.68 & 4. 49 & 4.97 & 4. 54 & 4. 67 \\
\hline & $21: 00$ (6-step) & 9.37 & 8.42 & 10.03 & 8.71 & 9.13 \\
\hline & 22:00 (12-step) & 15.56 & 14.76 & 15.87 & 15. 62 & 15. 45 \\
\hline & 23:00 (18-step) & 22.87 & 20.37 & 20.42 & 10. 17 & 18. 46 \\
\hline & 24:00 (24-step) & 32.64 & 30.42 & 33. 17 & 30.93 & 31.79 \\
\hline
\end{tabular}

\subsubsection{Summary: Based on experiments I-III}

Based on the above two experiments, we draw the following conclusions:

- The developed combined model based on linear and nonlinear features can provide effective wind speed forecasting results with higher accuracy and strong stability, so it can be widely used in actual application.

- The combined model can take full use of the advantages of each model and make each individual forecasting model perform better in certain cases, which can effectively forecast future wind speed compared with the individual models.

- A modified optimization algorithm, AFSA-ACO, is developed in this paper to optimize the weight of each individual model, which can further improve the forecasting performance of the developed model.

- The combined model including two linear forecasting models and four nonlinear forecasting models, is successfully employed to separately capture the inherent feature of linear and nonlinear characteristics contained in wind speed and ultimately results in success. Thus, it has a superior forecasting ability and will be widely used in the forecasting fields. 


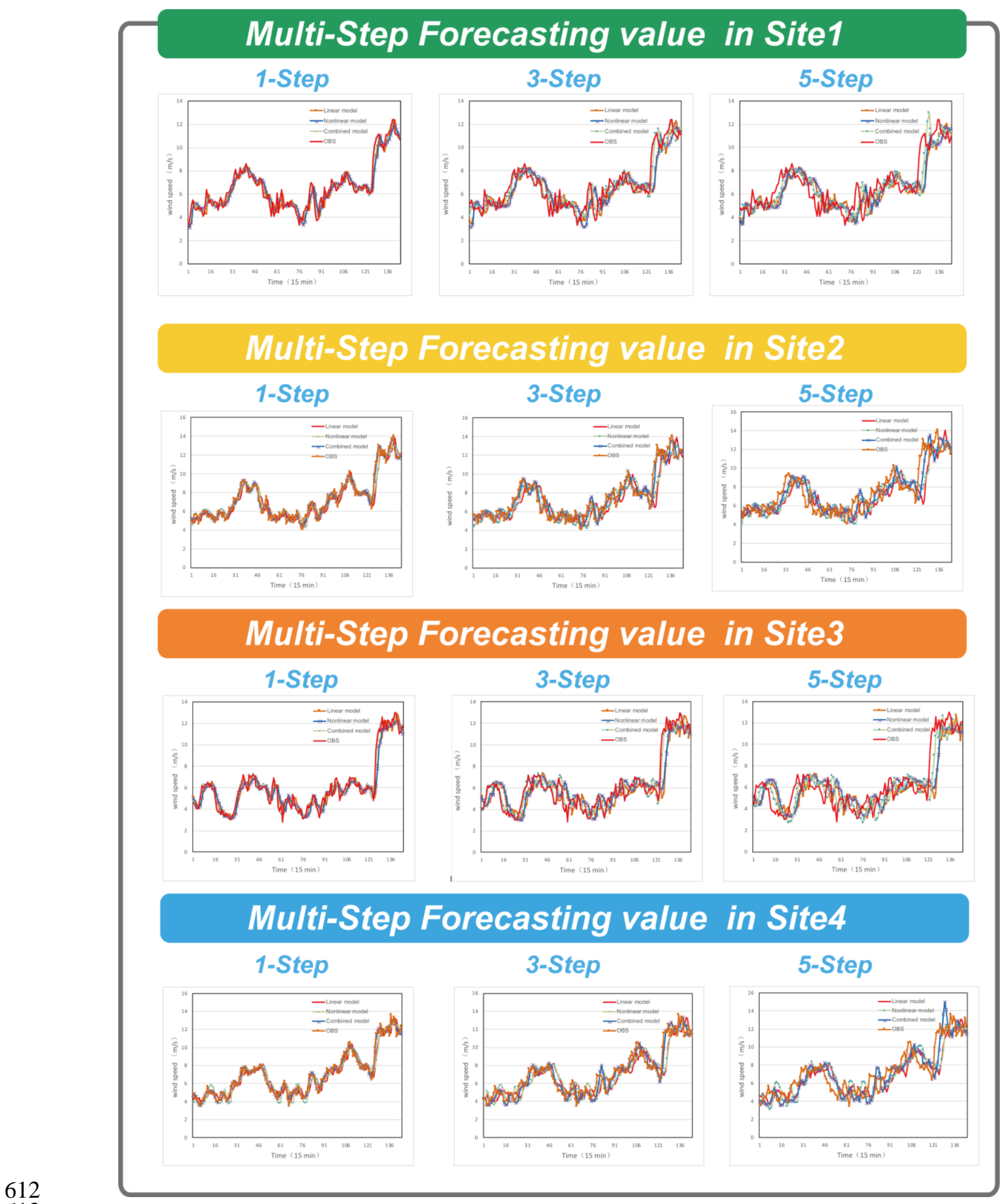

613

614

Fig. 5. Forecasted values of four models. 
Table 15 Typical results of the combined model and the results of the other models

\begin{tabular}{|c|c|c|c|c|c|c|c|c|c|c|c|c|}
\hline Model & \multicolumn{3}{|c|}{ Test with 1-step } & Average & \multicolumn{3}{|c|}{ Test with 3-step } & Average & \multicolumn{3}{|c|}{ Test with 5-step } & Average \\
\hline & \multicolumn{12}{|c|}{ MAE } \\
\hline BP & 0.3073 & 0.3574 & 0.3229 & 0.3292 & 0.3823 & 0.4117 & 0.3756 & 0.3899 & 0.5723 & 0.5542 & 0.5871 & 0.5712 \\
\hline ENN & 0.5144 & 0.4874 & 0.5331 & 0.5116 & 0.5830 & 0.7538 & 0.6421 & 0.6596 & 0.7491 & 0.8426 & 0.8857 & 0.8258 \\
\hline GRNN & 0.4611 & 0.4862 & 0.4792 & 0.4755 & 0.7126 & 0.6857 & 0.6643 & 0.6875 & 0.8651 & 0.9311 & 1.0635 & 0.9532 \\
\hline WNN & 0.5857 & 0.5671 & 0.5362 & 0.5630 & 0.7634 & 0.8214 & 0.7963 & 0.7937 & 0.8269 & 0.8869 & 1.0326 & 0.9155 \\
\hline ARIMA & 0.2972 & 0.3642 & 0.3971 & 0.3528 & 0.4286 & 0.5542 & 0.4831 & 0.4886 & 0.8631 & 0.9017 & 0.8517 & 0.8722 \\
\hline ES & 6230 & 0.6541 & 0.5628 & 0.6133 & 0.8542 & 0.9423 & 0.7988 & 0.8651 & 1.1354 & 1.0823 & 1.4529 & 1.2235 \\
\hline \multirow[t]{2}{*}{ Combined } & 0.3157 & 0.3627 & 0.3129 & 0.3304 & 0.3942 & 0.4217 & 0.3544 & 0.3901 & 0.6133 & 0.5648 & 0.5219 & 0.5667 \\
\hline & \multicolumn{12}{|c|}{ MAPE } \\
\hline BP & 4.55 & 4.86 & 4.71 & 4.71 & 5.82 & 6.27 & 5.64 & 5.91 & 8.87 & 8.41 & 9.12 & 8.80 \\
\hline ENN & 7.42 & 6.93 & 7.12 & 7.16 & 8.62 & 9.74 & 8.63 & 9.00 & 13.42 & 13.22 & 13.75 & 13.46 \\
\hline GRNN & 6.24 & 6.37 & 6.42 & 6.34 & $10 . .11$ & 9.27 & 8.94 & 9.44 & 11.47 & 11.86 & 12.73 & 12.02 \\
\hline WNN & 10.11 & 9.43 & 8.79 & 9.44 & 10.29 & 11.57 & 11.36 & 11.07 & 12.57 & 12.86 & 14.07 & 13.17 \\
\hline ARIMA & 4.86 & 5.18 & 5.72 & 5.25 & 7.09 & 7.24 & 6.93 & 7.09 & 13.57 & 14.26 & 13.01 & 13.61 \\
\hline ES & 10.37 & 10.54 & 9.84 & 10.25 & 12.94 & 13.54 & 12.37 & 12.95 & 16.89 & 15.74 & 18.42 & 17.02 \\
\hline \multirow[t]{2}{*}{ Combined } & 4.39 & 4.93 & 4.12 & 4.48 & 5.39 & 5.61 & 5.24 & 5.41 & 8.62 & 8.35 & 7.96 & 8.31 \\
\hline & \multicolumn{12}{|c|}{ MSE } \\
\hline BP & 0.1411 & 0.1721 & 0.1645 & 0.1592 & 0.2119 & 0.2327 & 0.2047 & 0.2164 & 0.6742 & 0.5680 & 0.6849 & 0.6424 \\
\hline ENN & 0.4673 & 0.5132 & 0.4865 & 0.4890 & 0.6714 & 0.8216 & 0.7468 & 0.7466 & 1.3514 & 1.1967 & 1.2532 & 1.2671 \\
\hline GRNN & 0.2762 & 0.2886 & 0.2931 & 0.2860 & 0.8453 & 0.7810 & 0.7246 & 0.7836 & 1.2357 & 1.3392 & 1.4531 & 1.3427 \\
\hline WNN & 0.9263 & 0.8446 & 0.7597 & 0.8435 & 0.9582 & 1.1052 & 1.0596 & 1.0410 & 1.3987 & 1.3547 & 1.5017 & 1.4184 \\
\hline ARIMA & 0.1571 & 0.1669 & 0.1764 & 0.1668 & 0.3129 & 0.4186 & 0.3562 & 0.3626 & 1.3619 & 1.4358 & 1.2956 & 1.3644 \\
\hline ES & 0.8741 & 0.8452 & 0.7599 & 0.8264 & 1.2576 & 1.3017 & 1.2294 & 1.2629 & 2.1047 & 1.9351 & 2.5033 & 2.1810 \\
\hline Combined & 0.1475 & 0.1582 & 0.1463 & 0.1507 & 0.2071 & 0.2483 & 0.1984 & 0.2179 & 0.6420 & 0.5939 & 0.5781 & 0.6047 \\
\hline
\end{tabular}

616

617 
Table 16 Results of the bias-variance framework regarding the combined model and other models

\begin{tabular}{|c|c|c|c|c|c|c|c|}
\hline \multicolumn{8}{|c|}{ Site 1} \\
\hline & Combined model & BPNN & ENN & WNN & GRNN & ARIMA & $\mathrm{ES}$ \\
\hline Bias & -37.2472 & 40.8293 & 208.24407 & 154.46983 & 110.4698 & 94.2908 & 156.09009 \\
\hline Variance & $2.56186 * 10^{6}$ & $2.23397 * 10^{6}$ & $5.57679 * 10^{6}$ & $4.67358 * 10^{6}$ & $3.78945 * 10^{6}$ & $3.65556 * 10^{6}$ & $3.68538 * 10^{6}$ \\
\hline \multicolumn{8}{|c|}{ Site 2} \\
\hline & Combined model & BPNN & ENN & WNN & GRNN & ARIMA & $\mathrm{ES}$ \\
\hline Bias & -26.0644 & 39.0858 & -150.1512 & -73.7867 & -131.0260 & -87.39465 & -138.86687 \\
\hline Variance & $1.78499 * 10^{5}$ & $2.94337 * 10^{6}$ & $6.79984 * 10^{5}$ & $3.37324 * 10^{5}$ & $4.49748 * 10^{6}$ & $3.82120 * 10^{5}$ & $4.84572 * 10^{5}$ \\
\hline \multicolumn{8}{|c|}{ Site 3} \\
\hline & Combined model & BPNN & ENN & WNN & GRNN & ARIMA & ES \\
\hline Bias & -29.8109 & 47.5106 & 209.0871 & 71.9564 & -102.2839 & -57.0692 & 128.0058 \\
\hline Variance & $2.02388 * 10^{6}$ & $2.65826 * 10^{6}$ & $7.10694 * 10^{6}$ & $3.87078 * 10^{6}$ & $4.30894 * 10^{6}$ & $3.12246 * 10^{6}$ & $4.14463 * 10^{6}$ \\
\hline \multicolumn{8}{|c|}{ Site 4} \\
\hline & Combined model & BPNN & ENN & WNN & GRNN & ARIMA & ES \\
\hline Bias & 31.1363 & -48.1986 & -117.1073 & -117.7205 & -100.2213 & 54.8568 & 134.0037 \\
\hline Variance & $2.57589 * 10^{5}$ & $3.34525 * 10^{6}$ & $6.06825 * 10^{5}$ & $4.57947 * 10^{5}$ & $3.71257 * 10^{5}$ & $3.19408 * 10^{5}$ & $3.72514 * 10^{5}$ \\
\hline
\end{tabular}




\section{Discussion}

An insightful discussion about the developed model is conducted in this section, including the improvements of the developed model, the significance of the developed model, the effectiveness of the combined strategy and the computation time.

\subsection{The improvements of the developed combined model}

To conduct a comprehensive analysis for the developed model, a metric, the decreased relative error of index $\left(D R E_{\text {index }}\right)$ [30], is employed in this paper to provide the improvements of information of the developed combined model, as how much the $D R E_{\text {index }}$ is means how much promotion between the two compared model, which is defined as

$$
D R E_{\text {index }}=\frac{\text { index }_{i}-\text { index }_{j}}{\text { index }_{i}}
$$

In this paper, we take the MAPE index as an illustration. The detailed results are presented in Table 18. According to Table 18, the developed combined model shows a great improvement compared with the other models. For example, for one-step forecasting, the developed combined model leads to improvements of $4.8832 \%$, $37.4302 \%, 29.3375 \%, 52.5424 \%, 14.6667 \%$ and $56.2927 \%$ in terms of the average MAPE compare with BPNN, ENN, GRNN, WNN, ARIMA and ES, respectively. The maximum decreased error of the average MAPE is $58.2239 \%$, and the minimum is $4.8832 \%$. Thus, the developed combined forecasting model is superior to other compared models and can achieve a satisfactory and effective forecasting performance when it is applied in engineering applications.

\begin{tabular}{|c|c|c|c|c|c|c|c|c|c|}
\hline \multirow{2}{*}{ Times } & \multicolumn{3}{|c|}{ Combined model vs. BPNN } & \multicolumn{3}{|c|}{ Combined model vs. ENN } & \multicolumn{3}{|c|}{ Combined model vs. GRNN } \\
\hline & 1-step & 3-step & 5-step & 1-step & 3-step & 5-step & & & \\
\hline 1 & & & & & & & & & \\
\hline 2 & & & & & & & & & \\
\hline 3 & & & & & & & & & \\
\hline & & & & 37.4302 & & & & & \\
\hline \multirow{2}{*}{ Times } & \multicolumn{3}{|c|}{ Combined model vs. WNN } & \multicolumn{3}{|c|}{ Combined model vs. ARIMA } & \multicolumn{3}{|c|}{ Combined model vs. ES } \\
\hline & & & & & & & & & \\
\hline 1 & 56.5 & & & & & & & & \\
\hline 2 & & & & & & & & & \\
\hline 3 & & & & & & & & & \\
\hline Average & 52.5424 & 51.1292 & 36.9021 & 14.6667 & 23.6953 & 38.9420 & 56.2927 & 58.2239 & 51.1751 \\
\hline
\end{tabular}

641

642

643

644

645

646

647

\subsection{The significance of the developed combined model}

In this section, a widely employed hypothesis testing approach, the Diebold-Mariano Test [31], is applied to evaluate the significance of the developed combined model with other comparison models. The specific theory of this method can be observed as follows.

Under a given a significance level, $\alpha$, the null hypothesis, $H_{0}$ represents that there is no significant difference between the proposed model and the compared 
model in terms of their forecasting performance. In contrast, $H_{1}$ is different from $H_{0}$. The related formulas can be written as

$$
\left\{\begin{array}{l}
H_{0}: E\left[l\left(e_{i}^{1}\right)\right]=E\left[l\left(e_{i}^{2}\right)\right] \\
H_{1}: E\left[l\left(e_{i}^{1}\right)\right] \neq E\left[l\left(e_{i}^{2}\right)\right]
\end{array}\right.
$$

wherein $l$ is the loss function of the forecasting error and $e_{i}^{j}, j=1,2$ indicates the prediction errors of the comparison models and the proposed model.

Moreover, the DM test statistics can be described as:

$$
D M=\frac{\sum_{i=1}^{n}\left(l\left(e_{i}^{1}\right)-l\left(e_{i}^{2}\right)\right) / n}{\sqrt{S^{2} / n}} s^{2}
$$

where $s^{2}$ is an estimation for the variance of $D_{i}=l\left(e_{i}^{1}\right)-l\left(e_{i}^{2}\right)$.

Finally, under the given the significance level $\alpha$, are employed to compared with the, if the DM statistic

If the computed values of DM fall outside the interval $\left[-Z_{\alpha / 2}, Z_{\alpha / 2}\right]$, the null hypothesis $H_{0}$ will be rejected. This case means that there is no significant difference in the forecasting performance between the proposed model and the compared model. Otherwise, the null hypothesis will be accepted.

Table 19 Diebold-Mariano test between the comparison models and the proposed model

\begin{tabular}{cccc}
\hline DM test & 1-step & 3-step & 5-step \\
\hline BPNN & $1.8236541^{* * *}$ & $1.7334923^{* * *}$ & $1.9230125^{* * *}$ \\
ENN & $2.2239706^{* *}$ & $2.2854941^{* *}$. & $2.3680649^{* * *}$ \\
GRNN & $1.7582973^{* * *}$ & 1.6247201 & $1.8496255^{* * *}$ \\
WNN & $2.1593657^{* *}$ & $2.2064192^{* *}$ & $2.4723091^{* *}$ \\
ARIMA & $2.2453762^{* *}$ & $2.3981643^{* *}$ & $2.5491064 * *$ \\
ES & $6.8723623^{*}$ & $7.12681234^{*}$ & $7.2790154^{*}$ \\
\hline
\end{tabular}

$*$ is the $1 \%$ significance level; $* *$ is the $5 \%$ significance level; $* * *$ is the $10 \%$ significance level;

From Table 19, it can be easily observed that the values of $|\mathrm{DM}|$ are all much greater than $Z_{0.05}=1.645$. Finally, we conclude that the null hypothesis can be accepted $\mathrm{T}$ the $10 \%$ significance level, which also means there is a $90 \%$ probability that the alternative hypothesis will be accepted. This case shows that the proposed combined method has a significant difference with the comparison approaches with the significance level $90 \%$. In other words, our proposed combined model exhibits a significant improvement in performance compare with other models.

\subsection{The effectiveness of the combined strategy of the developed combined model}

To verify the effectiveness of the combined strategy based on TCM-NNCT and weight-coefficient optimization, a comparison between the developed combined model and the combined model based on the simple average strategy is conducted in this section. The comparison results are shown in Table 20, which shows that the developed combined model shows excellent performance compared with the combined model with the simple average strategy. Therefore, we can draw the conclusion that the developed combined model, which is based on TCM-NNCT and 
680 the modified optimization algorithm, AFSA-ACO, effectively improves the 681 performance for wind speed forecasting.

682 To further evaluate the optimization capacity of the proposed combined model, 683 several common combined models, which based on different weight-coefficient 684 optimization algorithms (BFGS, PSO and ACO), are used to compare with the 685 developed combined model. Table 21 lists the MAE, MAPE and MSE values of 686 four combined models, which are optimized by the BFGS, PSO, ACO and 687 AFSA-ACO algorithms form four sites. The MAE, MAPE and MSE values of the 688 developed model are less than the other combined models in most cases, which 689 indicates that the combined model optimized by the AFSA-ACO algorithm is 690 relatively more accurate than the other combined models that optimized by BFGS, 691 PSO and ACO, respectively. Thus, it can be concluded that compared with other 692 combined models, the combined modes based on AFSA-ACO can provide more 693 accurate wind-speed forecasting. 
Table 20 Comparison results of different combined strategies

\begin{tabular}{|c|c|c|c|c|c|c|c|c|c|c|}
\hline \multirow{2}{*}{ Sites } & \multirow{2}{*}{ Combined Strategy } & \multicolumn{3}{|c|}{ MAE } & \multicolumn{3}{|c|}{ MAPE } & \multicolumn{3}{|c|}{ MSE } \\
\hline & & 1-step & 3-step & 5-step & 1-step & 3-step & 5-step & 1-step & 3-step & 5-step \\
\hline \multirow[t]{2}{*}{ Site 1} & Simple average strategy & 0.4648 & 0.6207 & 0.8353 & 7.26 & 8.95 & 12.80 & 0.4737 & 0.7096 & 1.3544 \\
\hline & Developed model & 0.3157 & 0.3942 & 0.6133 & 4.39 & 5.39 & 8.62 & 0.1475 & 0.2071 & 0.6420 \\
\hline \multirow[t]{2}{*}{ Site 2} & Simple average strategy & 0.4816 & 0.6949 & 0.8665 & 7.22 & 9.61 & 12.73 & 0.4718 & 0.7768 & 1.3049 \\
\hline & Developed model & 0.3627 & 0.4217 & 0.5648 & 4.93 & 5.61 & 8.35 & 0.1582 & 0.2483 & 0.5939 \\
\hline \multirow[t]{2}{*}{ Site 3} & Simple average strategy & 0.4719 & 0.6267 & 0.9789 & 7.10 & 8.98 & 13.52 & 0.4400 & 0.7202 & 1.4486 \\
\hline & Developed model & 0.3129 & 0.3544 & 0.5219 & 4.12 & 5.24 & 7.96 & 0.1463 & 0.1984 & 0.5781 \\
\hline \multirow[t]{2}{*}{ Site 4} & Simple average strategy & 0.4931 & 0.6832 & 0.9231 & 7.28 & 9.35 & 12.91 & 0.4824 & 0.7548 & 1.4275 \\
\hline & Developed model & 0.3276 & 0.4157 & 0.6014 & 4.97 & 5.51 & 8.27 & 0.1607 & 0.2293 & 0.6371 \\
\hline
\end{tabular}

695

696

697

698

699

700

701

702 
Table 21 Comparison results of different combined models

\begin{tabular}{|c|c|c|c|c|c|c|c|c|c|c|c|c|}
\hline \multirow{3}{*}{$\begin{array}{c}\text { Model } \\
\text { BFGS-CM }\end{array}$} & \multicolumn{3}{|c|}{ Test with 1-step } & \multirow{3}{*}{$\begin{array}{c}\text { Average } \\
0.3640\end{array}$} & \multicolumn{3}{|c|}{ Test with 3-step } & \multirow{3}{*}{$\begin{array}{l}\text { Average } \\
0.4294\end{array}$} & \multicolumn{3}{|c|}{ Test with 5-step } & \multirow{3}{*}{$\begin{array}{c}\text { Average } \\
0.6858\end{array}$} \\
\hline & \multicolumn{9}{|c|}{ 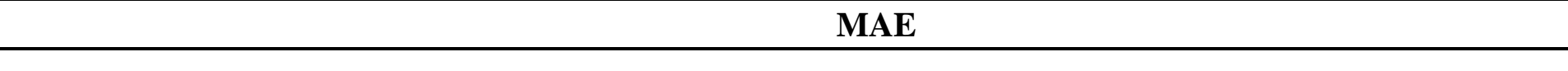 } & & & \\
\hline & 0.3624 & 0.3953 & 0.3342 & & 0.4298 & 0.4679 & 0.3905 & & 0.7231 & 0.6874 & 0.6469 & \\
\hline PSO-CM & 0.3871 & 0.4102 & 0.3571 & 0.3848 & 0.4531 & 0.5016 & 0.4176 & 0.4574 & 0.6574 & 0.6152 & 0.5826 & 0.6184 \\
\hline $\mathrm{ACO}-\mathrm{CM}$ & 0.4239 & 0.4436 & 0.4258 & 0.4311 & 0.5182 & 0.5583 & 0.5247 & 0.5337 & 0.8018 & 0.7513 & 0.7041 & 0.7524 \\
\hline \multirow[t]{2}{*}{ Pro. CM } & 0.3157 & 0.3627 & 0.3129 & 0.3304 & 0.3942 & 0.4217 & 0.3544 & 0.3901 & 0.6133 & 0.5648 & 0.5219 & 0.5667 \\
\hline & \multicolumn{12}{|c|}{ MAPE } \\
\hline BFGS-CM & 4.68 & 4.72 & 4.33 & 4.58 & 5.77 & 6.13 & 5.69 & 5.86 & 9.14 & 8.83 & 8.24 & 8.74 \\
\hline PSO-CM & 4.86 & 5.26 & 4.51 & 4.88 & 5.92 & 6.35 & 6.02 & 6.10 & 9.48 & 9.16 & 8.87 & 9.17 \\
\hline $\mathrm{ACO}-\mathrm{CM}$ & 5.10 & 5.39 & 4.87 & 5.12 & 6.58 & 7.18 & 6.24 & 6.67 & 9.72 & 9.33 & 9.04 & 9.36 \\
\hline \multirow[t]{2}{*}{ Pro. CM } & 4.39 & 4.93 & 4.12 & 4.48 & 5.39 & 5.61 & 5.24 & 5.41 & 8.62 & 8.35 & 7.96 & 8.31 \\
\hline & \multicolumn{12}{|c|}{ MSE } \\
\hline BFGS-CM & 0.1533 & 0.1562 & 0.1497 & 0.1531 & 0.2971 & 0.3416 & 0.2691 & 0.3026 & 0.6839 & 0.5847 & 0.6012 & 0.6233 \\
\hline PSO-CM & 0.1569 & 0.1613 & 0.1524 & 0.1569 & 0.3479 & 0.4017 & 0.2903 & 0.3466 & 0.7103 & 0.7482 & 0.6861 & 0.7149 \\
\hline $\mathrm{ACO}-\mathrm{CM}$ & 0.1822 & 0.1884 & 0.1763 & 0.1823 & 0.4107 & 0.4425 & 0.3876 & 0.4136 & 0.8482 & 0.7930 & 0.7425 & 0.7946 \\
\hline Pro. CM & 0.1475 & 0.1582 & 0.1463 & 0.1507 & 0.2071 & 0.2483 & 0.1984 & 0.2179 & 0.6420 & 0.5939 & 0.5781 & 0.6047 \\
\hline
\end{tabular}




\subsection{The computation time of the developed combined model and comparisons}

Table 22 shows the average computational time for each model at different sites. The responses of BPNN, ENN, GRNN, WNN, ARIMA, ES and the developed combined model are $5.56 \mathrm{~s}, 6.42 \mathrm{~s}, 7.13 \mathrm{~s}, 6.42 \mathrm{~s}, 312.46 \mathrm{~s}, 3.17 \mathrm{~s}$ and $17.75 \mathrm{~s}$, respectively. Among the models, the developed combined model has the second longest computation time, and the ES model has the shortest time. The computation time of each model is within the acceptable range. Although the combined model has the longest computation time, it is superior to all of the compared models. Therefore, the developed combined model is both short and applicable for predicting the wind speed in wind power plants, it can be used in engineering application.

Table 22 The computation time of each model

\begin{tabular}{cccc}
\hline Model & Computation time (s) & Model & Computation time (s) \\
\hline BPNN & 5.56 & ARIMA & 312.46 \\
ENN & 6.42 & ES & 3.17 \\
GRNN & 7.13 & Combined model & 17.75 \\
WNN & 6.42 & & \\
\hline
\end{tabular}

\section{Conclusion}

Accurate wind speed forecasting plays a vital role in the operation and management of wind power grids, which can bring considerable benefits and increase the utilization rate of wind energy. Thus, it is highly desirable to develop an optimized wind speed forecasting model with a high forecasting accuracy. However, individual forecasting models cannot always meet the increasing requirements. More specifically, individual forecasting models have some limitations and cannot perform forecasting effectively under certain conditions. The combined model can make full use of the merits of each model and can effectively forecast future wind speeds compared with the individual models. In this paper, a novel combined forecasting model based on a modified version of the AFSA and ACO, the AFSA-ACO, with two linear models, i.e., ES and ARIMA, and four nonlinear methods, i.e., BPNN, GRNN, WNN and ENN, was successfully proposed. More specifically, to enhance the forecasting capacity of the proposed combined model, the modified version of the AFSA and ACO, the AFSA-ACO, was integrated into this model and employed to optimize the weight of each individual model. There are two forecasting parts, the linear and nonlinear parts, for enhancing the forecasting performance, and they are used to separately capture the inherent linear and nonlinear features contained in wind speed. The case studies reveal that the developed combined model performs better than the other compared models, is an effective and promising technique for wind speed forecasting and can improve the forecasting effectiveness by combining these two linear and four nonlinear algorithms. Furthermore, based on a series of insightful discussions of our case studies, the effectiveness of the developed combined model is proven to be significantly superior to the compared models. For example, the DM test results reveals that the developed combined model has a higher degree accuracy than and is a significantly different from the other benchmark models. Despite the effectiveness of using the novel combined model illustrated above, some limitations certainly exist. For example, only wind speed series with 10-min intervals were employed to assess the performances of the developed model in this paper. Further research could make forecast using hourly, monthly and quarterly wind-speed data. Moreover, for future 
745

746

747

748 Acknowledgements: This work was supported by the National Natural Science 749 Foundation of China 41630421.

research, the proposed combined model can be employed in other forecasting fields, such as electricity price, air quality, traffic flow and stock index forecasting.

750 


\section{References}

752 [1] L. Martin, Wind Energy - The Facts: A Guide to the Technology, Economics doi:10.1016/j.jclepro.2010.02.016.

[2] A. Kumar, K. Kumar, N. Kaushik, S. Sharma, S. Mishra, Renewable energy in India: Current status and future potentials, Renew. Sustain. Energy Rev. 14 (2010) 2434-2442. doi:10.1016/j.rser.2010.04.003.

[3] C. Monteiro, H. Keko, R. Bessa, V. Miranda, A quick guide to wind power forecating: state-of-the-art 2009., (2009).

[4] J. Yan, Y. Liu, S. Han, Y. Wang, S. Feng, Reviews on uncertainty analysis of wind power forecasting, Renew. Sustain. Energy Rev. 52 (2015) 1322-1330. doi:10.1016/j.rser.2015.07.197.

[5] S. Qin, F. Liu, J. Wang, Y. Song, Interval forecasts of a novelty hybrid model for wind speeds, Energy Reports. 1 (2015) 8-16. doi:10.1016/j.egyr.2014.11.003.

[6] G. Giebel, R. Brownsword, G. Kariniotakis, M. Denhard, C. Draxl, The State-Of-The-Art in Short-Term Prediction of Wind Power A Literature Overview, Tech. Report, ANEMOS.plus. (2011) 1-109. papers://6470de79-5287-45a9-8e4f-b629919aff7a/Paper/p5443.

[7] S. Al-Yahyai, Y. Charabi, A. Gastli, Review of the use of numerical weather prediction (NWP) models for wind energy assessment, Renew. Sustain. Energy Rev. 14 (2010) 3192-3198. doi:10.1016/j.rser.2010.07.001.

[8] A.K. Rajeevan, P.. Shouri, U. Nair, ARIMA Based Wind Speed Modeling for Wind Farm Reliability Analysis and Cost Estimation, J. Electr. Eng. Technol. 11 (2016) 869-877. doi:10.5370/JEET.2016.11.4.869.

[9] M.G. De Giorgi, A. Ficarella, M.G. Russo, Short-term wind forecasting using artificial neural networks (ANNs), in: Energy Sustain. Ii, 2009: pp. 197-208. doi:10.2495/esu090181.

[10] Z. Guo, J. Wu, H. Lu, J. Wang, A case study on a hybrid wind speed forecasting method using BP neural network, Knowledge-Based Syst. 24 (2011) 1048-1056. doi:10.1016/j.knosys.2011.04.019.

[11] C. Yu, Y. Li, M. Zhang, Comparative study on three new hybrid models using Elman Neural Network and Empirical Mode Decomposition based technologies improved by Singular Spectrum Analysis for hour-ahead wind speed forecasting, Energy Convers. Manag. 147 (2017) 75-85. doi:10.1016/j.enconman.2017.05.008.

[12] W. Zhang, Z. Qu, K. Zhang, W. Mao, Y. Ma, X. Fan, A combined model based on CEEMDAN and modified flower pollination algorithm for wind speed forecasting, Energy Convers. Manag. 136 (2017) 439-451. doi:10.1016/j.enconman.2017.01.022.

[13] J. Wang, J. Heng, L. Xiao, C. Wang, Research and application of a combined model based on multi- objective optimization for multi-step ahead wind speed forecasting, Energy. 125 (2017) 591-613. doi:10.1016/j.energy.2017.02.150.

[14] L. Xiao, J. Wang, Y. Dong, J. Wu, Combined forecasting models for wind energy forecasting: A case study in China, Renew. Sustain. Energy Rev. 44 (2015) 271-288. doi:10.1016/j.rser.2014.12.012.

[15] Y. Yang, Y. Chen, Y. Wang, C. Li, L. Li, Modelling a combined method based on ANFIS and neural network improved by DE algorithm: A case study for short-term electricity demand forecasting, Appl. Soft Comput. (2016). doi:10.1016/j.asoc.2016.07.053. 
[16] R. Rahmani, R. Yusof, M. Seyedmahmoudian, S. Mekhilef, Hybrid technique of ant colony and particle swarm optimization for short term wind energy forecasting, J. Wind Eng. Ind. Aerodyn. 123 (2013) 163-170. doi:10.1016/j.jweia.2013.10.004.

[17] T. Sen, H.D. Mathur, A new approach to solve Economic Dispatch problem using a Hybrid ACO-ABC-HS optimization algorithm, Int. J. Electr. Power Energy Syst. 78 (2016) 735-744. doi:10.1016/j.ijepes.2015.11.121.

[18] M.K. Patel, M.R. Kabat, C.R. Tripathy, A hybrid ACO/PSO based algorithm for QoS multicast routing problem, Ain Shams Eng. J. 5 (2014) 113-120. doi:10.1016/j.asej.2013.07.005.

[19] H. Hassani, Singular Spectrum Analysis: Methodology and Comparison, J. Data Sci. 5 (2007) 239-257. doi:10.3189/172756506781828863.

[20] G.E.P. Box, G.M. Jenkins, G.C. Reinsel, Time Series Analysis: Forecasting \& Control, 1994. doi:10.1016/j.ijforecast.2004.02.001.

[21] D. Asteriou, S.G. Hall, ARIMA Models and the Box-Jenkins Methodology, Appl. Econom. (2011) 265-286. doi:10.1002/(SICI)1099-131X(199705)16:3<147::AID-FOR652>3.0.CO;2-X.

[22] D.E. Rumelhart, G.E. Hinton, R.J. Williams, Learning representations by back-propagating errors, Nature. 323 (1986) 533-536. doi:10.1038/323533a0.

[23] D.F. Specht, A General Regression Neural Network, IEEE Trans. Neural Networks. 2 (1991) 568-576. doi:10.1109/72.97934.

[24] J. Wang, W. Zhang, Y. Li, J. Wang, Z. Dang, Forecasting wind speed using empirical mode decomposition and Elman neural network, Appl. Soft Comput. 23 (2014) 452-459. doi:10.1016/j.asoc.2014.06.027.

[25] J.M. Bates, C.W.J. Granger, The Combination of Forecasts, J. Oper. Res. Soc. 20 (1969) 451-468. doi:10.1057/jors.1969.103.

[26] V. Maniezzo, A. Colorni, The ant system applied to the quadratic assignment problem, IEEE Trans. Knowl. Data Eng. 11 (1999) 769-778. doi:10.1109/69.806935.

[27] M. Dorigo, M. Birattari, T. Stutzle, Ant colony optimization, IEEE Comput. Intell. Mag. 1 (2006) 28-39. doi:10.1109/MCI.2006.329691.

[28] P. Jiang, F. Liu, Y. Song, A hybrid forecasting model based on date-framework strategy and improved feature selection technology for short-term load forecasting, Energy. 119 (2017) 694-709. doi:10.1016/j.energy.2016.11.034.

[29] P. Du, J. Wang, Z. Guo, W. Yang, Research and application of a novel hybrid forecasting system based on multi-objective optimization for wind speed forecasting, Energy Convers. Manag. 150 (2017) 90-107. doi:10.1016/j.enconman.2017.07.065.

[30] W. Yang, J. Wang, R. Wang, Research and application of a novel hybrid model based on data selection and artificial intelligence algorithm for short term load forecasting, Entropy. 19 (2017). doi:10.3390/e19020052.

[31] F.X. Diebold, R.S. Mariano, Comparing Predictive Accuracy, J. Bus. Econ. Stat. 13 (1995) 253-263. doi:10.1080/07350015.1995.10524599. 